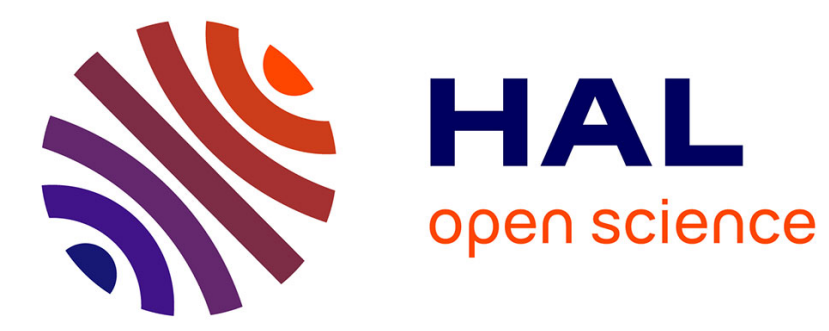

\title{
Local-Explosive Approximations to Null Distributions of the Johansen Cointegration Test, with an Application to Cyclical Concordance in the Euro Area
}

Guillaume Chevillon

\section{- To cite this version: \\ Guillaume Chevillon. Local-Explosive Approximations to Null Distributions of the Johansen Cointe- gration Test, with an Application to Cyclical Concordance in the Euro Area. 2012. hal-00751925}

\section{HAL Id: hal-00751925 \\ https://essec.hal.science/hal-00751925}

Preprint submitted on 14 Nov 2012

HAL is a multi-disciplinary open access archive for the deposit and dissemination of scientific research documents, whether they are published or not. The documents may come from teaching and research institutions in France or abroad, or from public or private research centers.
L'archive ouverte pluridisciplinaire HAL, est destinée au dépôt et à la diffusion de documents scientifiques de niveau recherche, publiés ou non, émanant des établissements d'enseignement et de recherche français ou étrangers, des laboratoires publics ou privés. 
Local-Explosive Approximations to Null Distributions of the Johansen

\title{
Cointegration Test,
}

with an Application to Cyclical

Concordance in the

Euro Area

\author{
Research Center \\ ESSEC Working Paper 1210 \\ 2012
}

Guillaume Chevillon 


\title{
Local-Explosive Approximations to Null Distributions of the Johansen Cointegration Test, with an Application to Cyclical Concordance in the Euro Area.
}

Guillaume Chevillon*

ESSEC Business School, Paris

October 12, 2012

\begin{abstract}
This paper considers approximating the finite sample null-distribution of a test statistic as its asymptotic distribution under a local alternative. We focus on the Likelihood Ratio test for the rank of cointegration and use nonlinearities that represent some finite sample distributional features. Reliable approximations are obtained using a class of locally explosive models. An empirical evaluation of the concordance of European business cycles through cointegration shows that some standard corrections lead to underestimating the number of cointegrating relations and induce volatile results.
\end{abstract}

JEL codes: C12, C32, E32.

Keywords: Cointegration, Finite samples, Local-asymptotics, Business Cycle Convergence.

*chevillon@essec.edu; I wish to thank and Michael Clements, Stéphane Grégoir, David Hendry, Bent Nielsen, Sophocles Mavroeidis and Robert Taylor for their valuable comments on an earlier version of the paper. I also thank participants at the NBER/NSF Time Series Conference, the European, Far Eastern and Latin American meetings of the Econometric Society, the Journée d'Econométrie at the University of Paris-Ouest, the ACE Conference in Hong Kong, and seminar participants at OFCE/Sciences-Po, ESSEC, CREST and Brown University. 


\section{Introduction and overview}

This paper analyzes a new application to the well established local-asymptotic framework that was introduced to econometrics by Bobkoski (1983), Cavanagh (1985), Phillips (1987), and Chan and Wei (1987). We study whether finite sample null distributions of test statistics can be approximated using asymptotic distributions under a local alternative. The reason we do so is twofold: local asymptotics provide $(i)$ an easily implementable method to generate distributions that is successful in approximating finite samples distributions (see Perron, 1991); and (ii) a simple way to induce some nonlinearities which we show to be relevant in finite samples and may not otherwise be taken into account (as they vanish using conventional asymptotics). In particular, this framework allows to model how the asymptotic similarity a test with respect to some parameters may not hold in finite samples. To our knowledge, the idea of using the distribution under the alternative as a finite sample approximation is new and we show that it works (at the cost of introducing nuisance parameters, that could be tabulated in practice). In considering distributions under the alternative to represent null distributions, we differ from the class of continuous record approximations studied in Perron (1991).

To clarify the procedure we follow, consider the Dickey-Fuller test of the null $H_{0}: \varphi=0$ vs $H_{1}: \varphi<0$ in

$$
\Delta y_{t}=\tau+\varphi y_{t-1}+\epsilon_{t}, \text { for } t=1, \ldots, T
$$

where $T^{-1 / 2} \sum_{t=1}^{\lceil r T\rceil} \epsilon_{t} \Rightarrow \sigma W(r)$, with $W$ a Wiener process. ${ }^{1}$ The deterministic com-

\footnotetext{
${ }^{1}$ Conventionally, $\Rightarrow$ denotes weak convergence of the associated probability measure on $D^{p}[0,1]$, $p \geq 1$, the space of $\mathbb{R}^{p}$-valued functions on the interval $[0,1]$ which are right continuous and have finite left limits. $\lceil x\rceil$ denotes the integer part of any real scalar $x . C^{p}[0,1]$ is the subspace of
} 
ponent $\tau$ implies a trend that is a linear under the null, $y_{t} \underset{{ }_{H_{0}}}{=} y_{0}+\tau t+\sum_{i=1}^{t} \epsilon_{i}$, but nonlinear under the alternative: $y_{t} \underset{\bar{H}_{1}}{\overline{=}}(1+\varphi)^{t} y_{0}+\tau \frac{1-(1+\varphi)^{t}}{-\varphi}+\sum_{i=1}^{t}(1+\varphi)^{t-i} \epsilon_{i}$. Asymptotically, the nonlinear trend vanishes under $H_{1}$ as $(1+\varphi)^{t} \rightarrow 0$. By contrast, if we consider the local alternative $H_{1}: \varphi=e^{\phi / T}-1, \phi \neq 0$, where $T$ is the sample size, then the nonlinear trend does not vanish asymptotically since $(1+\varphi)^{T} \rightarrow e^{\phi}$ as $T \rightarrow \infty .^{2}$ In this paper, we consider approximating the finite-sample distribution - under $H_{0}$ - of the $t$-statistic on $\varphi$ as that under the local alternative $H_{1}$. The distributions that we obtain may hence depend nonlinearly on deterministic parameters even when the test statistics are similar with respect to deterministic terms under $H_{0}$. In order to see the effect the trend coefficient, $\tau$ in expression (1) above, has on test statistics under $H_{1}$, we also model it as local asymptotic (a Pitman drift), in effect assuming $\tau=\psi / \sqrt{T}$ for $\psi \neq 0$ so $y_{T}=O_{p}(\sqrt{T})$. Contrary to, say, Elliott, Rothenberg and Stock (1996), we do not assume that $\tau=o\left(T^{-1 / 2}\right)$ so the deterministic trend remains present asymptotically and interacts with the stochastic trends as in e.g. Stock and Watson (1996) and Vogelsang (1998). In this context, $\psi$ matters for the test distributions even as $T \rightarrow \infty$.

We develop a multivariate extension of the framework above and consider the Johansen (1988) Likelihood Ratio (LR) test. The distribution of the test statistic under the null of $q$ linearly independent cointegration vectors for a system of $p$ variables satisfies asymptotically:

$-2 \log Q(H(q) \mid H(p)) \Rightarrow \operatorname{tr}\left\{\int_{0}^{1} d \mathbf{W}(r) \mathbf{F}^{\prime}(r)\left[\int_{0}^{1} \mathbf{F}(r) \mathbf{F}^{\prime}(r) d r\right]^{-1} \int_{0}^{1} \mathbf{F}(r) d \mathbf{W}^{\prime}(r)\right\}$, $D^{p}[0,1]$ of continuous functions.

${ }^{2}$ The nonlinear trend also remains under the alternative when modeling the trend as additive to the local asymptotic process: $y_{t}=\mu+\tau t+z_{t}$, with $z_{t}=e^{\phi / T} z_{t-1}+\epsilon_{t}$ leads to replacing $\tau$ and $\varphi$ with $\tau-\left(e^{\phi / T}-1\right)(\mu+\tau(t-1))$ and $e^{\phi / T}-1$ in expression (1). 
where $\mathbf{W}$ is a multivariate Brownian motion of dimension $p-q$ and $\mathbf{F}$ is a a function of $\mathbf{W}$ whose actual definition depends on the parametric specification of the model (see Johansen, 1991). Several authors have derived finite sample corrections of the LR statistic: see for instance Johansen (2000, 2002a and 2002b), Swensen (2006) and Cavaliere, Rahbek and Taylor (2012). The approximation we derive in this paper relies on local asymptotic alternatives to the null of cointegration, drawing in part on Pesavento (2004), both on the stationary and explosive sides. We show in particular that locally explosive processes can provide reliable finite sample approximations.

The main difference between our framework and existing approximations to the finite sample distribution the LR statistic is that previous authors have considered distributions that are similar with respect to the parameters of higher-order deterministic components (when a polynomial trend is present). This draws on the asymptotic similarity of versions of the LR statistic (see Johansen, 1994, and Nielsen and Rahbek, 2000) under the null. ${ }^{3}$ Here, working under the alternative generates nonlinear trends. This implies that, in the multivariate framework, the suggestion by Johansen (1994) to restrict the linear trend to lie within the cointegration space may not restore similarity with respect to the deterministic components. In addition we will show that some rotations are no longer possible in the presence of a localasymptotic drift, contrary to e.g. Pesavento (2004) and Saikkonen and Lütkepohl (1999).

Our results allows to revisit, inter alia, the methodologies of Enders (1988), Mac-

\footnotetext{
${ }^{3}$ Lütkepohl and Saikkonen (2000) have also proposed an LM test that is similar with respect to deterministic components, see also Saikkonen and Lütkepohl (2000a) and Saikkonen and Lütkepohl (2000b).
} 
Donald and Taylor (1991) and many authors since. We apply our theoretical results to the question of whether business cycles in the euro area exhibited concordance prior to the 2007 financial crisis. We restrict our attention to this early period as the recessive effect of the posterior financial crisis may blur the analysis and we hope that information about the early period of the introduction of the euro has some potential interest to the policy decisions that fiscal and monetary authorities will have to take in the near future. The problem of small sample size is then very acute and requires finite sample distributional approximations.

This paper is organized as follows. We first present in section 2 our proposed model of near cointegration for deriving finite sample approximations to the distributions. Section 3 derives the resulting distribution of the Likelihood Ratio test statistic. We proceed to a Monte Carlo analysis in section 4 where we validate the finite sample approximation. An empirical illustration follows in section 5 in which it appears that European economies converged in the run-up to the euro introduction and immediately started diverging thereafter. Proofs of the main results are presented in the appendix.

The paper uses the following notation: vectors are denoted by $\alpha=\left(\alpha_{1}: \ldots: \alpha_{p}\right)$, with $\alpha^{\prime}=\left(\alpha_{1}, \ldots, \alpha_{p}\right)$. For any $(p \times q)$ matrix $\alpha$ of full rank, we define $\alpha_{\perp}$ of dimension $p \times(p-q)$ such that $\left(\alpha, \alpha_{\perp}\right)$ is of full rank; we define the generalized projection operator $\bar{\alpha}=\alpha\left(\alpha^{\prime} \alpha\right)^{-1}$. Also, we denote by $\operatorname{diag}(\alpha, \beta, \ldots)$ the (block-)diagonal matrix with diagonal elements $(\alpha, \beta, \ldots)$. 


\section{The model}

Consider the usual autoregressive representation for analyzing cointegration in the $p$-dimensional vector of variables $\mathbf{x}_{t}$ (see Johansen, 1991):

$$
\Delta \mathbf{x}_{t}=\Pi \mathbf{x}_{t-1}+\Upsilon t^{n_{d}}+\sum_{i=0}^{n_{d}-1} \psi_{i} t^{i}+\epsilon_{t} \quad(t=1, \ldots, T)
$$

where $\epsilon_{t} \sim \operatorname{IN}[\mathbf{0}, \boldsymbol{\Omega}]$. If $\mathbf{x}_{t}$ is $\mathrm{I}(1)$ and $\boldsymbol{\Pi}$ is of reduced rank $q$, then there exist $\alpha$ and $\beta$ of dimension $(p \times q)$ such that $\boldsymbol{\Pi}=\alpha \beta^{\prime}$ and that $\beta^{\prime} \mathbf{x}_{t}-\mathbf{E}\left[\beta^{\prime} \mathbf{x}_{t}\right]$ is stationary. $\mathbf{x}_{t}$ cointegrates, with cointegration vector $\beta$. The test for the hypothesis $\boldsymbol{\Pi}=\alpha \beta^{\prime}$, and possibly $\Upsilon=\alpha \rho^{\prime}$, under Gaussian errors, is carried out as a LR test by the technique of reduced rank regression via a ratio, $Q(H(q) \mid H(p))$ defined below in expression (10), and whose asymptotic distribution for the null of $q \leq p$ cointegration relations under model (3) is derived in Johansen $(1991,1994)$ as expression (2) where $\mathbf{F}$ is function of a multivariate Brownian motion and whose actual form depends on $n_{d}$ and on whether $\alpha_{\perp}^{\prime} \Upsilon=\mathbf{0}$. We set the deterministic terms as $\mathbf{d}_{t}=(1, t)$ if $n_{d}=1$ and $\mathbf{d}_{t}=1$ if $n_{d}=0$, and collect them as $\mathbf{\Psi} \mathbf{d}_{t}=\mathbf{\Upsilon} t^{n_{d}}+\sum_{i=0}^{n_{d}-1} \psi_{i} t^{i}$. We have assumed without loss of generality and for ease of notation that lags of $\Delta \mathbf{x}_{t}$ do not enter equation (3); we assume also that $\mathbf{x}_{0}=\mathbf{0}$, although this is not an inconsequential assumption (see Müller and Elliott, 2003). The model implies that the vector process $\mathbf{x}_{t}$ admits the moving average representation:

$$
\mathbf{x}_{t}=\mathbf{C} \sum_{i=1}^{t}\left(\epsilon_{i}+\boldsymbol{\Psi} \mathbf{d}_{i}\right)+\mathbf{C}_{T}(L)\left(\epsilon_{t}+\boldsymbol{\Psi} \mathbf{d}_{t}\right)+\mathbf{A}
$$

where $L$ is the lag operator, $\mathbf{C}=\beta_{\perp}\left(\alpha_{\perp}^{\prime} \beta_{\perp}\right)^{-1} \alpha_{\perp}^{\prime}, \mathbf{A}$ is a stationary process and the power series for $\mathbf{C}_{T}(z)$ is convergent for $|z|<1+\delta$ for some $\delta>0$.

Consider now the local asymptotic model we use to derive distributional approx- 
imations. We define a triangular array $\mathbf{x}_{t, T}$, and modify (4) as follows:

$$
\mathbf{x}_{t, T}=\mathbf{C} \sum_{i=1}^{t} e^{\phi(t-i) / T}\left(\epsilon_{i}+\Psi \mathbf{B}_{T} \mathbf{d}_{i}\right)+\mathbf{C}_{T}(L)\left(\epsilon_{t}+\boldsymbol{\Psi} \mathbf{B}_{T} \mathbf{d}_{t}\right)+\mathbf{A}
$$

and

$$
\left\{\begin{array}{l}
\phi \in \mathbb{R} \\
\mathbf{B}_{T}=T^{-1 / 2} \operatorname{diag}\left(T^{0}, . ., T^{n_{d}}\right)^{-1} .
\end{array}\right.
$$

For notational ease, we omit the dependence of $\mathbf{x}_{t}$ in $T$ in the following. The moving average representation (5) implies an autoregressive representation (3) where deterministic components are damped by $\mathbf{B}_{T}$ and $\Pi=\alpha \beta^{\prime}$ is replaced with $\Pi=\alpha \beta^{\prime}+$ $\left(e^{\phi / T}-1\right) \mathbf{I}_{p} \cdot{ }^{4}$

The vector series generated by (5)-(6) is near integrated if $\alpha \beta^{\prime}$ is of reduced rank $q<p$, it is also near-cointegrated when $q>0$. In finite samples $\mathbf{x}_{t}$ is stationary. Trend stationarity asymptotically vanishes and the limit process is cointegrated, with cointegrating vector $\beta$. The choice of representation for locally cointegrated process is not unique: here, the process is trend stationary in finite samples; stochastic trends and cointegration only appear asymptotically. Other alternatives are possible, such as allowing stochastic trends at all sample sizes and sample-dependent cointegration rank as in Johansen (1995), chapter 14, or also in Elliott (1998) and Jansson and Haldrup (2002). Additionally, we restrict our attention for simplicity to a unique localizing parameter for the stochastic trend $\phi .{ }^{5}$ The distribution of $\mathbf{x}_{t}$ varies continuously for $\phi \rightarrow 0$. Let $T^{-1 / 2} \sum_{i=0}^{\lceil T r\rceil} \epsilon_{i} \Rightarrow \mathbf{\Sigma} \mathbf{W}(r)$, as $T \rightarrow \infty$, where $\mathbf{W}$ is a standard Brownian motion on $C^{p}[0,1]$ and $\boldsymbol{\Sigma}$ is a positive definite matrix such that $\boldsymbol{\Omega}=\boldsymbol{\Sigma} \boldsymbol{\Sigma}^{\prime}$. Under the local asymptotic framework above, if $\boldsymbol{\Psi} \neq \mathbf{0}$ the process exhibits a deterministic trend that is local since both its expectation and standard

\footnotetext{
${ }^{4}$ For simplicity we assume away lags of $\Delta \mathbf{x}_{t}$ in the right-hand side of expression (3).

${ }^{5}$ This could readily be extended to allow for a vector $\Phi=\left(\phi_{1}, \ldots, \phi_{p}\right)^{\prime}$.
} 
deviation are of the same order $O\left(T^{1 / 2}\right)$ : the process exhibits both stochastic and deterministic sources of non-stationarity and the two interact at all sample sizes and in the limit.

Local asymptotics have often be used in the context of unit roots for power analyses of tests (see e.g. Elliott, Müller and Watson, 2012) and for the construction of confidence intervals (from Stock, 1991, to Mikusheva, 2007). Local-to-zero drifts have been used inter alia in Monte Carlo simulations of unit root tests, first in Vogelsang (1998) and also in Rossi (2005) and Busetti and Harvey (2005); they have been analyzed as a model per se but in a univariate setting in Haldrup and Hylleberg (1995), Stock and Watson (1996), and also Nabeya and Sorensen (1994), but then, in the different context of continuous record asymptotics - as in this article to emphasize the interactions between stochastic and deterministic trends. They have also been used in Kim et al. (2004) who focus on trend stationary or difference stationary models, yet not on the presence of the two types of trends; but testing for the latter is the focus of Harvey et al. (2007). In a different representation, Kiviet and Phillips (2005) have proceeded to expansions of the unit root test statistics in powers of the drift and found that their approximations fared relatively badly for low drifts.

It should be noted that our purpose is not to provide a framework for cointegration testing that would be robust to local trends but simply to assess the properties of the LR test. Often a modeler is uncertain about the existence of a deterministic trend and wishes to perform a test for the rank of cointegration that is not affected by the presence of the absence of a trend (see e.g Lütkepohl and Saikkonen (2000)). Then she may use the union of two tests, one that is robust to some forms of trends, one that is to others, as in the multivariate extension, in Demetrescu et al. (2009), 
to the combination of several statistics that was proposed by Harvey et al. (2009).

To analyze the properties of locally trending processes, we define the Vasiček diffusion

$$
K_{\psi, \phi}^{\dagger}(r)=\psi f_{\phi}(r)+\int_{0}^{r} e^{\phi(r-s)} d W(s), \text { for } r \in[0,1]
$$

where the continuous deterministic functional $f_{(\cdot)}: \mathbb{R} \rightarrow C[0,1]$, with $\phi \in \mathbb{R} \backslash\{0\}$ is:

$$
f_{\phi}(\cdot): r \rightarrow \frac{e^{\phi r}-1}{\phi}
$$

and $f_{0}(r)=r$. By extension to (7), for a given $\sigma>0$, we denote by $K_{\psi, \phi}(r)$ the Gaussian diffusion $K_{\psi, \phi}(r)=\sigma K_{\psi / \sigma, \phi}^{\dagger}(r)$ with expectation $\psi f_{\phi}(r)$ and variance $\sigma^{2} f_{2 \phi}(r)$. It is standard in the financial literature to specify Vasiček diffusions as $K_{\psi \phi,-\phi}$. Hence, we refer instead to $K_{\psi, \phi}^{\dagger}(r)$ and $K_{\psi, \phi}(r)$ as trending OrnsteinUhlenbeck (TOU) processes.

A natural multivariate extension of the above uses the $n$-variate Ornstein-Uhlenbeck diffusion $\mathbf{J}_{\phi}^{\dagger}(r)=\int_{0}^{r} e^{\phi(r-s)} d \mathbf{W}(s)$ where

$$
T^{-1 / 2} \sum_{i=1}^{[T r]}\left(e^{\phi([T r]-i) / T} \epsilon_{i}\right) \Rightarrow \boldsymbol{\Sigma} \mathbf{J}_{\phi}^{\dagger}(r) \stackrel{\text { def }}{=} \mathbf{J}_{\phi}(r) .
$$

Multivariate TOU diffusions are defined as $\mathbf{K}_{\mathbf{\Psi}, \phi}^{\dagger}(r)=\mathbf{\Psi} \mathbf{f}_{\phi}(r)+\mathbf{J}_{\phi}^{\dagger}(r)$, with $\mathbf{f}_{\phi}(r)=\left(f_{\phi}(r), \int_{0}^{r} f_{\phi}(u) d u\right)^{\prime}$ for $n_{d}=1$; discarding the second element for $n_{d}=0 .{ }^{6}$ We also define $\mathbf{K}_{\boldsymbol{\Psi}, \phi}(r)=\mathbf{\Sigma} \mathbf{K}_{\boldsymbol{\Sigma}^{-1} \boldsymbol{\Psi}, \phi}(r)$.

\section{Inference}

In this section, we derive the distribution of the Likelihood Ratio test for the cointegration rank computed from model (3) using the corresponding asymptotic distri-

\footnotetext{
${ }^{6}$ To keep the notation readable, we omit the dependence of $\mathbf{f}_{\phi}$ on $n_{d}$ since it should always be clear to which we refer.
} 
bution when the data generating process (the DGP) is given by (5)-(6). By an abuse of language, we refer to the test as being similar with respect to some parameter under a given hypothesis (the null or the local alternative) when the distribution of its statistic does not depend on the value of the parameter under that hypothesis. Similarity is usually only referred to under the null, but since our aim is to approximate this distribution and because we only derive distributions under the alternative we hope that our message will be clear.

We first derive the asymptotic distribution of the scaled locally trending and locally cointegrated process in terms of the TOU diffusions.

Lemma 1 A locally cointegrated and locally trending process $\left\{\mathbf{x}_{t}\right\}$ which is generated by (5)-(6) satisfies:

$$
T^{-1 / 2} \mathbf{x}_{[T r]} \Rightarrow \mathbf{C K}_{\Psi, \phi}(r)
$$

The asymptotic distributions of the LR statistic under the null and local alternative depend on the model which is used, and especially on which deterministic components it contains (constants and trends).

We define the log likelihood ratio statistic that corresponds to model (3) as

$$
-2 \log Q(H(q) \mid H(p))=-T \sum_{i=q+1}^{p} \log \left(1-\widehat{\lambda}_{i}\right)
$$

where the eigenvalues $\lambda_{i}$ are estimated as solutions to the problem $\left|\lambda S_{11}-S_{10} S_{00}^{-1} S_{01}\right|=$ $|S(\lambda)|=0$, with $S_{i j}=T^{-1} \sum_{i=1}^{T} R_{i t} R_{j t}^{\prime}, R_{i t}=Z_{i t}-M_{i 2} M_{22}^{-1} Z_{2 t}, M_{i j}=T^{-1} \sum_{i=1}^{T} Z_{i t} Z_{j t}^{\prime}$, $Z_{0 t}=\Delta \mathbf{x}_{t}, Z_{1 t}=\mathbf{x}_{t-1}$ and $Z_{2 t}$ is made of the deterministic components that enter the model. We denote by $\mathrm{M}_{h}$ the model (4) with the following deterministic terms: $h=0$ in the absence of deterministic terms, $h=\mu$ when only a constant is included, $h=\tau$ with a constant and a linear trend. Models $\mathbf{M}^{*}$ and $\mathbf{M}_{\mu}^{*}$ refer to $\mathbf{M}_{\mu}$ and $\mathbf{M}_{\tau}$ 
with the highest order deterministic term that is restricted to lie in the cointegration space, i.e. $\boldsymbol{\Upsilon}=\alpha \rho^{\prime}$ in (3), so $Z_{1 t}$ is augmented with a constant $(h=\mu)$ or a linear trend $(h=\tau)$. The LR statistic computed according to model $\mathrm{M}_{h}^{m}, h \in\{0, \mu, \tau\}$ and $m \in\{\emptyset, *\}$, is denoted by $\operatorname{LR}_{h}^{m}$.

To derive the asymptotic distributions of the LR statistics computed as above, but where the DGP is $(5)$, we define $\mathbf{G}_{0}$ a $(p-q)$-variate TOU process given by

$$
\mathbf{G}_{0}=\mathbf{J}_{\phi}^{\dagger}+\left(\mathbf{0}_{1 \times p-q-1}, \mathbf{g}_{\phi}\right)^{\prime}
$$

where $\mathbf{g}_{\phi}(r)=\left(\bar{\tau}^{\prime} \mathbf{C} \boldsymbol{\Sigma} \boldsymbol{\Sigma}^{\prime} \mathbf{C}^{\prime} \bar{\tau}\right)^{-1 / 2} \bar{\tau}^{\prime} \mathbf{C} \boldsymbol{\Psi} \mathbf{f}_{\phi}(r) ; \mathbf{1}_{n_{d}+1}$ is a vector of ones of dimension $n_{d}+1$ and $\tau=\mathbf{C} \boldsymbol{\Psi} \mathbf{1}_{n_{d}+1}$. We extend the definition to $\mathbf{G}_{\mu}=\mathbf{G}_{0}-\int_{0}^{1} \mathbf{G}(r) d r$ and $\mathbf{G}_{\tau}=\mathbf{G}_{0}-a-b r$, where $a$ and $b$ are obtained by correcting $\mathbf{G}_{0}$ for a mean and a linear trend. Furthermore, we let $\mathbf{G}_{0}^{*}=\left(\mathbf{G}_{0}, 1\right)$ and $\mathbf{G}_{\mu}^{*}(r)=\left(\mathbf{G}_{\mu}(r), r\right)$. With the notations above, the following proposition holds.

Proposition 2 Assume that the data generating process is locally cointegrated and locally trending as generated by (5)-(6). The asymptotic distribution of the Likelihood Ratio statistic for the null of $q<p$ cointegration relations among $p$ variables using the standard approach of reduced rank regression in model $M_{h}, h \in\{0, \mu, \tau\}$ is given by:

$$
\mathrm{LR}_{h}^{m} \Rightarrow \operatorname{tr}\left\{\int_{0}^{1} d \mathbf{G}_{h}(r) \mathbf{G}_{h}^{\prime}(r)\left[\int_{0}^{1} \mathbf{G}_{h}(r) \mathbf{G}_{h}^{\prime}(r) d r\right]^{-1} \int_{0}^{1} \mathbf{G}_{h}(r) d \mathbf{G}_{h}^{\prime}(r)\right\}
$$

The asymptotic distribution of the LR test refers to the definition (11) which arises as $\mathbf{G}_{0}=\left(\alpha_{\perp}^{\prime} \mathbf{C} \boldsymbol{\Omega} \mathbf{C}^{\prime} \alpha_{\perp}\right)^{-1 / 2} \mathbf{H}$, where $T^{-1 / 2} \bar{\alpha}_{\perp}^{\prime} \mathbf{x}_{[T r], T} \Rightarrow \mathbf{H}$; hence $\mathbf{G}_{0}$ corresponds the standardized asymptotic distribution of a linear combination of the scaled common trends $\alpha_{\perp}^{\prime} \mathbf{x}_{t}$. Proposition 2 shows that inference drawn from the LR test may be affected by the coefficients of the deterministic components when 
these are allowed to interact asymptotically with the stochastic trend. Indeed, when $\phi=0, \mathbf{G}_{\tau}$, corrected for a constant and a linear trend, simplifies to $\mathbf{W}$, i.e. without deterministic terms. But for $\phi \neq 0$, the nonlinear function $\mathbf{f}_{\phi}(r)$ cannot be offset by a linear trend correction. Hence, under this setting, the asymptotic distribution depends upon $\mathbf{g}_{\phi}(r)$, i.e. it is not similar (under the local alternative) with respect to the projection of $\boldsymbol{\Psi}$ onto $s p\left(\alpha_{\perp}\right)$. More precisely, the distribution in proposition 2 depends on the ratio $\left(\bar{\tau}^{\prime} \mathbf{C} \boldsymbol{\Sigma} \boldsymbol{\Sigma}^{\prime} \mathbf{C}^{\prime} \bar{\tau}\right)^{-1 / 2} \bar{\tau}^{\prime} \mathbf{C} \boldsymbol{\Psi}$, with $\tau=\mathbf{C} \boldsymbol{\Psi} \mathbf{1}_{n_{d}+1}$, which writes $\psi / \sigma$ in univariate unit-root testing. The ratio $\boldsymbol{\Sigma}^{-1} \boldsymbol{\Psi}$ is commonly encountered when accounting for finite sample deviations from asymptotic distributions (see Kiviet and Phillips, 2005) so the result is comparable here. The definition of $\mathbf{G}_{0}$ implies that all deterministic components which belong to the space spanned by $\alpha$ have no influence on the distribution of the LR statistic, as in Nielsen and Rahbek (2000) and in the following proposition.

Proposition 3 Under the assumptions of proposition 2. Consider the hypothesis of a deterministic term restricted to the cointegrating space, as in $\mathrm{M}_{\mu}^{*}$. The LR test statistic satisfies under the local alternative:

$$
\mathrm{LR}_{\mu}^{*} \Rightarrow \operatorname{tr}\left\{\int_{0}^{1} d \mathbf{G}_{0}(r) \overline{\mathbf{G}}^{\prime}(r)\left[\int_{0}^{1} \overline{\mathbf{G}}(r) \overline{\mathbf{G}}^{\prime}(r) d r\right]^{-1} \int_{0}^{1} \overline{\mathbf{G}}(r) d \mathbf{G}_{0}^{\prime}(r)\right\} .
$$

where $\overline{\mathbf{G}}$ is $\left(\mathbf{G}_{0}, r\right)$ corrected for the mean.

Under $\mathrm{M}_{\mu}^{*}$, the distribution of the test statistic, under the local alternative, is asymptotically independent of the coefficients of $\boldsymbol{\Psi}$ that belong to $\operatorname{sp}(\alpha)$. Hence, under the local alternative, the test is not asymptotically similar with respect to deterministic terms if $\alpha_{\perp}^{\prime} \boldsymbol{\Psi} \neq \mathbf{0}$, contrary to the distribution under the null, as in Johansen (1994). Denoting $\mathbf{\Psi}_{\mathbf{t}}=\boldsymbol{\Psi}_{0}+\boldsymbol{\Psi}_{1}$ t, the distribution of $\mathrm{LR}_{\mu}^{*}$ asymptotically depends on $\boldsymbol{\Psi}_{0}$ if $\alpha_{\perp}^{\prime} \boldsymbol{\Psi}_{0} \neq \mathbf{0}$ (for the same reasons of nonlinearities arising from 
the local-asymptotic setting, as in the discussion to the previous proposition). This means that, even when appropriately restricting the linear trend to the cointegrating space, the LR test statistic may depend - under the local alternative - on the value of the deterministic components. The most closely related study, by Nielsen and Rahbek (2000) considered only the case $\alpha_{\perp}^{\prime} \boldsymbol{\Psi}_{0}=\mathbf{0}$ in finite samples, but there are no reasons to assume that both the trend and the intercept that appear in expression (3) should be restricted a priori to the cointegrating space. An unrestricted constant is a regular feature of cointegrated vector equilibrium correction models and its presence is advocated in empirical modeling: see inter alia the recommended models in Johansen (1995), page 81, and Juselius (2006) section 6.3.

Proposition 3 helps to see that, for model (5) with $n_{d}=1$ the only situation where asymptotic similarity of the LR statistic holds under the local alternative is when there is no quadratic trend in the data, the constant and linear trends are entered unrestrictedly in model (5) and if $\phi=0$ : this is under the null. This differs from the results in Pesavento (2004) where the linearity of the trend is maintained under the alternative so it can be eliminated by the rotation suggested in Johansen (1994).

We have shown in this section that even when the deterministic components are correctly modeled under the null, the value of their coefficients may actually matter for inference under the local alternative. It remains to be seen whether the latter accurately reflects the finite sample distributions so that they indeed constitute nuisance parameters. 


\section{Monte Carlo}

\subsection{Experimental design}

We validate the locally trend approximation via a Monte Carlo experiment where we simulate integrals via samples of 20,000 discrete observations, and use 20,000 replications of the processes. In the simulations, we set $\boldsymbol{\Omega}=\mathbf{I}_{p}$, and let, for a cointegration $\operatorname{rank} q$, the vectors $\beta=\left(\mathbf{I}_{q}, \mathbf{0}_{q \times(p-q)}\right)^{\prime}$ and $\alpha=-\beta$ where $\mathbf{I}_{q}$ is the $q$ dimensional unit matrix and $\mathbf{0}_{m \times n}$ a $(m \times n)$-matrix of zeros. Then $\boldsymbol{\Pi}=\operatorname{diag}\left(-\mathbf{I}_{q}, \mathbf{0}_{(p-q) \times(p-q)}\right)$ so the process consists of $q$ white noise processes and $(p-q)$ pure random walks. We choose $\beta_{\perp}=\left(\mathbf{0}_{(p-q) \times q}, \mathbf{I}_{(p-q)}\right)^{\prime}$ and

$$
\alpha_{\perp}=[(p-q) \psi]^{-1}\left[\begin{array}{cc}
\mathbf{0}_{q \times(p-q-1)} & \mathbf{0}_{q \times 1} \\
\mathbf{I}_{(p-q-1)} & \mathbf{1}_{(p-q-1)} \\
\mathbf{0}_{1 \times(p-q-1)} & 1
\end{array}\right]
$$

with $\mathbf{1}_{(p-q)}$ a vector of ones of dimension $p-q$. Hence $\mathbf{C}=\beta_{\perp}\left(\alpha_{\perp}^{\prime} \beta_{\perp}\right)^{-1} \alpha_{\perp}^{\prime}=$ $\operatorname{diag}\left(\mathbf{0}_{q \times q}, \mathbf{I}_{(p-q)}\right)$. We record only a few results here, focusing on $n_{d}=0$ with $\boldsymbol{\Psi}=(\psi, 0, \ldots)^{\prime} ;$ hence $\alpha_{\perp}^{\prime} \boldsymbol{\Psi} \neq \mathbf{0}$ and $\mathbf{G}_{0}(r)=\mathbf{J}_{\phi}^{\dagger}(r)+\left(0, \ldots, 0,(p-q)^{1 / 2}|\psi| f_{\phi}(r)\right)^{\prime}$. In the asymptotic distribution of the LR test under the local alternative, the impact of the drift is then multiplied by the square root of the number of common stochastic trends: $(p-q)^{1 / 2}$.

\subsection{Simulation results}

In order to analyze the quality of the local-asymptotic approximation to finite sample distributions we report three types of results depending on whether the underlying model is correctly specified for the deterministic terms. 
First, we assess in table 1 whether the LR tests for the number of cointegration relations are robust to misspecified local non-zero drifts in finite samples. ${ }^{7}$ This studies whether the presence of low nonzero trends matter for inference on the cointegration rank. For this we report quantiles of the LR statistic for model $M_{\mu}$ where the data is generated as in subsection 4.1. For comparison we also report the Ahn-Reinsel finite sample correction, see Ahn and Reinsel (1999) and Cheung and Lai (1993). Here the model is misspecified for $\psi \neq 0$ and we see that the presence of a small unmodelled trend may flaw inference on the rank of cointegration when few stochastic trends are present. The role played by the number of stochastic trends shows that when only a few are present, the deterministic components may impact the distributions as in Evans and Savin (1984). This motivates the use of local deterministic trends to allow their coefficients to have an impact on asymptotic distributions under the local alternatives.

Second, we investigate whether the local asymptotic setting accurately describes finite sample behavior: tables $2,3,4$ and 5 present the $j$-percentiles, $\mathrm{x}_{j}$, of the simulated finite sample distribution of the Likelihood Ratio test for the presence of $q$ cointegrating relations, i.e. $p-q$ common stochastic trends, where the models considered are respectively $\mathrm{M}_{0}, \mathrm{M}_{\mu}, \mathrm{M}_{\mu}^{*}$ and $\mathrm{M}_{\tau}$. The finite sample distributions are compared to those obtained under the local asymptotic framework by means of a nonparametric test for the equality of the distributions. In the absence of closed form expression for either distribution, we use the Mann and Whitney (1947) test for equality of distributions between two independent samples (each of 20,000 observations here). Under the null of distributional equality, the Mann-Whitney

\footnotetext{
${ }^{7}$ We report here the case of very small samples with $T=25$ observations to stretch the analysis to the limiting case studied in the empirical application.
} 
(noted MW henceforth) statistic asymptotically follows a standard Normal. The corresponding $p$-values are reported as functions of the near-integration parameter $\phi$. The figures in bold in the tables denote the values of $\phi$ that provide the best approximation, in terms $p$-values of the MW statistics, among those considered.

A first result emerges from most parameter combinations: the finite sample distributions of the Dickey-Fuller unit root tests (in the Wald, $t^{2}$, form when $p=1$, $q=0)$ or of one stochastic trend $(p-q=1)$ tend to be best approximated setting $\phi=0$ and, according to the MW test, the local asymptotic distribution accurately represents the finite sample behavior. Nabeya and Sorensen (1994) found similar results in the univariate unit root test. Notable exceptions to the former features are $\mathrm{LR}_{\mu}^{*}$ for samples of $T=50$ or 100 observations in which case $\phi=1$ performs better; also to some extent $\mathrm{LR}_{\mu}$ in which case $\phi=.25$ is better.

In the context of testing for the presence of several stochastic trends, it appears overall that using distributions under locally-explosive roots is increasingly beneficial when there are more trends. As regards the effect of the sample size, the patterns differ according to whether models are, or not, underparameterized for the deterministic components: in tables 2 and 3 where no deterministic trend is allowed under the null, using distributions with $\phi>0$ is relevant in very small samples and can lead to very good distributional approximations when $T=50$. Samples of 200 observations are better represented via smaller localizing parameters $(\phi \leq 0.25) ; \phi$ needs be larger for smaller sample sizes. By contrast, tables 4 and 5 show that the approximations are not accurate enough at $T=50$ (although allowing for $\phi>0$ still performs better than $\phi \leq 0)$.

Finally, we assess whether the local asymptotic framework accounts for the distribution of the test for a model that is well specified under the null. For this, we 
complement table 5 with figure 1 which records the distributions of $\mathrm{LR}_{\tau}$ from finite sample simulations and from the local asymptotic approximation. The skewed shape of the distribution for $p-q=1$ is well approximated letting $\phi=0$. As the number of stochastic trends $p-q$ increases, the distribution of $\mathrm{LR}_{\tau}$ resembles a Normal. Figure 2 presents the QQ plots of the proposed approximation $(\phi=.25)$ against quantiles of the simulated finite sample $\mathrm{LR}_{\tau}$. This corroborates the validity of our framework; in particular the QQ plots are closer than the MW statistic would imply. It must be noted that MW, as other tests with the purpose of comparing empirical distributions (such as Kolmogorov-Smirnov or Cramer-von Mises), tends to over-reject in very large samples. Unreported simulations show that this is especially the case for $p-q=1$, i.e. when the distribution is then further away from Gaussianity. Hence, we should be relatively confident about the quality of the proposed local-asymptotic approximation, even when the reported $p$-values are low.

In conclusion to the Monte Carlo, we should stress that the finite sample dependence of the test statistic on the value of the deterministic trend component is exemplified by table 5 where the MW test shows that the value of $\psi$ has an impact on the distributions.

\section{Cointegration among continental European GDP series}

We apply our methodology to assessing the degree of business cycle concordance between five continental European economies. Harmonization of business cycles is crucial for monetary policy to be timely in the whole currency area. Yet, the inception of the European Monetary Union - in 1999 - is still recent, and techniques 
relying on frequency analyses lack sufficient data for assessing the degree of cycle convergence. The finite sample analysis we develop in this paper therefore finds a natural application since GDP series are often considered to exhibit deterministic trends (see e.g. King et al., 1991). We restrict our attention to the observations prior to 2007 , i.e. before the recent financial crisis. The reason for this decision is that the recession induces concordance that is exceptional and blurs inference. It seems more interesting for fiscal and monetary authorities to see whether the concordance held under unexceptional times.

To assess the importance of considering the deterministic parameters when accounting for cointegration in finite samples, we test for the number of cointegrating relations among Gross Domestic Product (GDP) quarterly series ${ }^{8}$ of five major continental European economies: France (FR), Germany (GE), Italy (IT), the Netherlands (NL) and Spain (SP), as displayed on figure 3. The variables we consider clearly present a deterministic trend (which accounts inter alia for the growth of population and per capita productivity). The presence of significant cointegrating vectors is seen here as tantamount to some degree of concordance among the business cycles of these economies that altogether represent about $85 \%$ of the combined GDP of the euro area (first 11 members). The methodology we use is standard in the literature on economic convergence, see e.g. Enders (1988), Bernard and Durlauf (1995), and Manning (2002); it has in particular been used to assess the degree of convergence among European economies, as in MacDonald and Taylor (1991) and

\footnotetext{
${ }^{8}$ Datasets and replication files are available from the author. The series are taken from the OECD Economic Outlook. GDP is in volume at market prices. Series for Germany prior to 1991 are backcasts using the growth rate of Western Germany. Computations were performed using Ox and Oxmetrics. Panels are referred to as $(a)$ to $(d)$ from left to right, top to bottom.
} 
Brada et al. (2005). We do not use the term "convergence" since we do not test for a change in the cointegration rank but prefer the word concordance here. A higher rank of cointegration is viewed a higher degree of concordance. Convergence and divergence can be loosely analyzed through the variations in the $p$-values associated with test statistics.

The presence of stochastic trends is assessed via sequential testing for the rank $q$ of cointegration, starting from zero and increasing until a value is not rejected. We only compare the $p$-values computed from our proposed local-asymptotic distributions to those of Johansen (2002a) despite the existence of several alternative finite sample approximation techniques; the reason is that none of the existing approximations, such as that of Swensen (2006) and Cavaliere el al. (2012) account for the values of the deterministic parameters.

We present on figure 4 the $p$-values corresponding to the Likelihood Ratio test statistics under an $\operatorname{VAR}(1)$ assumption ${ }^{9}$ with a linear trend that is restricted (model $\mathbf{M}_{\mu}^{*}$ in panel $\left.(a)\right)$, or not $\left(\mathbf{M}_{\tau}\right.$ in panel $\left.(b)\right)$, to lie in the cointegrating space. The $p$ values are computed using the finite sample Bartlett correction in Johansen (2002a). The sample size is allowed to vary, from $T=25$ to 146 where the endpoint is held at $2006 q 4$ and the origin varies backwards from $2000 q 4$ to $1970 q 3$. The figures report on the horizontal axis as the origin of the sample. We compare the $p$-values in figure 4 to those obtained from the local-asymptotic distributions proposed in the paper using different values of $\phi$ and $\psi$. In figure 5, the local-asymptotic distributions are computed by Monte Carlo: setting $(\phi, \psi)=(0.5,0)$ with 20,000 replications. We then relax the assumption that $\psi=0$ and compute the distributions using a

\footnotetext{
${ }^{9}$ This is the model that was chosen by sequential likelihood ratio testing of a $\operatorname{VAR}(k)$ against a $\operatorname{VAR}(k+1)$, starting with a $\operatorname{VAR}(4)$.
} 
parametric bootstrap, i.e. Monte Carlo simulation (20,000 replications) of the finite sample distributions using an estimated $\psi$ parameter. The parametric bootstrap relies on simulating the distribution of the nonpivotal statistic conditional on the realization of the estimator of a nuisance parameter. This method has been shown inter alia by Davidson and MacKinnon (1999) to perform well. ${ }^{10}$ Here, distributions are conditioned on the estimates of local drift parameters $\psi$ from univariate regressions: we regress the variables on a linear trend and set $\widehat{\psi}$ as maximizing the ratio of the linear trend coefficient over the estimated standard deviation of the detrended residuals. Figures 6 and 7 report the resulting recursive $p$-values for models $\mathrm{M}_{\mu}^{*}$ and $\mathrm{M}_{\tau}$ respectively. Finally, we complement the previous figures with figure 8 which reports the recursively selected rank of cointegration using a significance level of $10 \%$.

Inference drawn from the result reported in the figures vary. The $p$-values computed using the Johansen (2002a) technique are much more volatile than using the other methods. In figure 4 and under both $\mathrm{M}_{\mu}^{*}$ and $\mathrm{M}_{\tau}$, the null of no-cointegration exhibits very high $p$-values (above 70\%) when using data prior to the the first oil crisis of September 1973. These $p$-values exhibit a sharp decrease when the sample starts in late 1973 or later. For a sample origin between 1974 and 1981, the $p$-values oscillate below $40 \%$ and stay briefly below $10 \%$ under model $\mathrm{M}_{\mu}^{*}$ in several occasions. Economic growth drops below trend in several European economies in 1981-1982 following the second oil shock and disinflation policies. Both test statistics indicate that for samples starting between 1981 and 1995, the presence of one cointegration relation is not rejected among these GDP variables (despite slight divergence

\footnotetext{
${ }^{10}$ Chan, Park and Song (2006) use a nonparametric bootstrap for the distribution of cointegration tests in a different framework.
} 
following the 1987Q3 Stock Market crash under $\mathrm{M}_{\mu}^{*}$ ). Under model $\mathrm{M}_{\mu}^{*}$ divergence seems to start around 1993 when the UK was forced to withdraw from the European Exchange Rate Mechanism (ERM) and the subsequent increase in the fluctuation margins. The same pattern is observable under model $\mathrm{M}_{\tau}$ for the $p$-values of nulls of $q>0$, but the null of no-cointegration is only non-rejected starting in 1996, with a brief relapse for sample origins around the introduction of the euro. To summarize, the tests under the Johansen finite sample approximation mostly show no cointegration except during the 1981-1995 period, but the $p$-values may exhibit large variations when the sample size changes. Inference hence appears partly unreliable.

We now compare the previous results with those under the local approximations reported in figures 5-7. With the approximation under a local alternative, the $p$ values and conclusions are more stable. Yet they differ according to which of $\mathrm{M}_{\mu}^{*}$ and $\mathrm{M}_{\tau}$ is considered. We focus our analysis on $\mathrm{M}_{\tau}$ since it is robust to the position of the deterministic trends with respect to the cointegration space. The tests and distributions lead to conclude to the presence of two cointegration relations throughout the sample, with the apparition of a third when considering samples spanning only post-1990 data. It is through this third cointegration relation that we discuss the convergence and divergence of the economies considered. The $p$-values for the null that $q \leq 2$ are very close to unity when considering samples that either contain data from the 1970s or that span only post-2000 data. Figure 7 in particular is most instructive in that it clearly shows that European economies converged in the run-up to euro qualification but that they immediately started diverging following its introduction in 1999. The actual $p$-values reported in the figure correspond to averages over several regimes (convergence/divergence). Hence, their actual levels may be misleading, since we do not consider rolling windows of observations, though 
their dynamics is not. In light of our Monte Carlo results from section 4, the most convincing reported results are in 7 , panels $(b)$ and $(c)$.

In conclusion, discarding the information content of the deterministic parameters leads to under-estimating the dimension of the cointegration space. Figure 8 shows that whereas using the distribution in Johansen (2002a), a modeler infers the presence of almost no cointegrating relations (at most one) amongst the five European economies considered, this does not hold when taking the value of the deterministic trend parameter into account: the degree of concordance among the European business cycles is tested to be lower and much more volatile using Johansen (2002a) than according to the framework proposed here.

\section{Conclusions}

This paper shows that finite sample null distributions of test statistics can be approximated using asymptotic distributions under a local alternative. Although the local asymptotic distributions are commonly used to assess the power of tests, the application we propose seems new in the literature. We show how the methodology can be used to model finite sample interactions between multivariate deterministic components and stochastic trends and we apply it to approximating the Johansen (1988) likelihood ratio test for the rank of cointegration.

We show that the approximating distribution of the test may depend on the parameters of the deterministic components that enter the data generating process. Simulations indicate that finite sample distributions tend to be better approximated via a locally explosive stochastic behavior. This is in particular the case when the model allows for more than one stochastic trends under the null. 
In an empirical application to the estimation of the number of cointegration vectors among the GDP series of five continental European economies, it is shown that the finite sample approximation technique of Johansen (2002a) may lead to underestimating the dimension of the cointegrating space; it also leads to a higher degree of volatility in the reported inference results. The technique we propose provides sharper finite sample results on the degree of concordance among the business cycles of continental Europe. It is found empirically that the economies started diverging around the introduction of the euro.

\section{References}

Ahn, S. K. and C. G. Reinsel (1990). The influence of VAR dimension on estimator biases. Econometrica 67, 163-181.

Bernard, A. B. and S. N. Durlauf (1995). Convergence in international output. Journal of Applied Econometrics 10(2), 97-108.

Bobkoski, M. (1983). Hypothesis testing in nonstationary time series. Unpublished PhD thesis, Dept. of Statistics, University of Wisconsin, Madison.

Brada, J. C., A. M. Kutan, and S. Zhou (2005). Real and monetary convergence between the European Union's core and recent member countries: A rolling cointegration approach. Journal of Banking and Finance 29(1), 249 - 270.

Busetti, F. and A. Harvey (2005). Testing for trend. mimeo, University of Cambridge.

Cavanagh, C. (1985). Roots local to unity. Unpublished manuscript, Harvard University. 
Chan, N. H. and C. Z. Wei (1987). Asymptotic inference for nearly nonstationary AR(1) processes. Annals of Statistics 15(3), 1050-1063.

Chang, Y., J. Y. Park, and K. Song (2006). Bootstrapping cointegrating regressions. Journal of Econometrics 133(2), 703-39.

Cheung, Y.-W. and K. S. Lai (1993). Finite-sample sizes of Johansen's Likelihood Ratio tests for cointegration. Oxford Bulletin of Economics and Statistics 55, $313-28$.

Davidson, R. and J. G. MacKinnon (1999). The size distortion of bootstrap tests. Econometric Theory 15(3), 361-76.

Demetrescu, M., H. Lütkepohl, and P. Saikkonen (2009). Testing for the cointegrating rank of a vector autoregressive process with an uncertain deterministic trend term. Econometrics Journal 12(3), 414-435.

Elliott, G. (1998). On the robustness of cointegration methods when regressors almost have unit roots. Econometrica 66, 149-58.

Elliott, G., U. Müller, and M. W. Watson (2012). Nearly optimal tests when a nuisance parameter is present under the null hypothesis. Working paper, Princeton University.

Elliott, G., T. J. Rothenberg, and J. H. Stock (1996). Efficient tests for an autoregressive unit root. Econometrica 64(4), 813-36.

Enders, W. (1988). Arima and cointegration tests of PPP under fixed and flexible exchange rate regimes. The Review of Economics and Statistics 70(3), 504-508. 
Evans, G. B. and N. E. Savin (1984). Testing for unit roots: 2. Econometrica 52, $1241-69$.

G. CAVALIERE, A. R. and A. M. R. TAYLOR (2012). Bootstrap determination of the co-integration rank in vector autoregressive models. Econometrica 80(4), $1721-40$.

Haldrup, N. and S. Hylleberg (1995). A note on the distribution of the least squares estimator of a random walk with drift: Some analytical evidence. Economics Letters 48, 221-8.

Hamilton, J. D. (1994). Time Series Analysis. Princeton, NJ: Princeton University Press.

Harvey, D., S. Leybourne, and R. Taylor (2007). A simple, robust and powerful test of the trend hypothesis. Journal of Econometrics 141, 1302-30.

Harvey, D. I., S. J. Leybourne, and A. M. R. Taylor (2009). Unit root testing in practice: dealing with uncertainty over the trend and initial condition (with commentaries and rejoinder). Econometric Theory 25, 587-636.

Jansson, M. and N. Haldrup (2002). Regression theory for nearly cointegrated time series. Econometric Theory 18(06), 1309-1335.

Johansen, S. (1988). Statistical analysis of cointegrating vectors. Journal of Economic Dynamics and Control 12, 231-254. Reprinted in R.F. Engle and C.W.J. Granger (eds.), Long-run Economic Relationships, Readings in Cointegration, Oxford University Press (1991). 
Johansen, S. (1991). Estimation and hypothesis testing of cointegration vectors in Gaussian vector autoregressive models. Econometrica 59, 1551-80.

Johansen, S. (1994). The role of the constant and linear terms in cointegration analysis of nonstationary time series. Econometric Reviews 13, 205-31.

Johansen, S. (1995). Likelihood-based inference in cointegrated vector auto-regressive models. Oxford: Oxford University Press.

Johansen, S. (2000). A bartlett correction factor for tests on the cointegrating relations. Econometric Theory 16(5), 740-78.

Johansen, S. (2002a). A small sample correction for the test of cointegrating rank in the vector autoregressive model. Econometrica $70(5), 1929-61$.

Johansen, S. (2002b). A small sample correction for the tests of hypotheses on the cointegrating vectors. Journal of Econometrics 111(2), 195-221.

Juselius, K. (2006). The cointegrated VAR model. Oxford: Oxford University Press.

Kim, T.-H., S. J. Leybourne, and P. Newbold (2004). Asymptotic mean-squared forecast error when an autoregression with linear trend is fitted to data generated by an I(0) or I(1) process. Econometric Theory 25(4), 583-602.

King, R., C. Plosser, J. Stock, and M. Watson (1991). Stochastic trends and economic fluctuations. American Economic Review 81(4), 819-40.

Kiviet, J. F. and G. D. A. Phillips (2005). Moment approximation for least-squares estimators in dynamic regression models with a unit root. Econometrics Journal 8(2), 115-142. 
Lütkepohl, H. and P. Saikkonen (2000). Testing for the cointegrating rank of a var process with a time trend. Journal of Econometrics 95, 177-98.

MacDonald, R. and M. P. Taylor (1991). Exchange rates, policy convergence, and the European Monetary System. The Review of Economics and Statistics 73(3), $553-558$.

Mann, H. B. and D. R. Whitney (1947). On a test of whether one of two random variables is stochastically larger than the other. Annals of Mathematical Statistics 18, 50-60.

Manning, N. (2002). Common trends and convergence? South East Asian equity markets, 1988-1999. Journal of International Money and Finance 21(2), 183 202.

Mikusheva, A. (2007). Uniform inference in autoregressive models. Econometrica $75(5), 1411-1452$.

Müller, U. K. and G. Elliott (2003). Tests for unit roots and the initial condition. Econometrica 71, 1269-86.

Nabeya, S. and B. Sorensen (1994). Asymptotic distributions of the least-squares estimators and test statistics in the near unit root model with non-zero initial value and local drift and trend. Econometric Theory 10, 937-66.

Nielsen, B. and A. Rahbek (2000). Similarity issues in cointegration models. Oxford Bulletin of Economics and Statistics 62, 5-22.

Perron, P. (1991). A continuous time approximation to the unstable first-order 
autoregressive process: The case without an intercept. Econometrica 59(1), 211236.

Pesavento, E. (2004). Analytical evaluation of the power of tests for the absence of cointegration. Journal of Econometrics 122, 349-84.

Phillips, P. C. B. (1987). Towards a unified asymptotic theory for autoregression. Biometrika $74(3), 535-547$.

Rossi, B. (2005). Testing long-horizon predictive ability with high persistence, and the meese-rogoff puzzle. International Economic Review 46(1), 61-92.

Saikkonen, P. and H. Lütkepohl (1999). Local power of likelihood ratio tests for the cointegrating rank of a var process. Econometric Theory 15, 50-78.

Saikkonen, P. and H. Lütkepohl (2000a). Testing for the cointegrating rank of a var process with an intercept. Econometric Theory 16, 373-406.

Saikkonen, P. and H. Lütkepohl (2000b). Trend adjustment prior to testing for the cointegrating rank of a var process. Journal of Time Series Analysis 21, 435-56.

Stock, J. H. (1991). Confidence intervals for the largest autoregressive root in u.s. macroeconomic time series. Journal of Monetary Economics 28(3), 435-459.

Stock, J. H. and M. W. Watson (1996). Confidence sets in regressions with highly serially correlated regressors. mimeo, Princeton University.

Swensen, A. R. (2006). Bootstrap algorithms for testing and determining the cointegration rank in var models. Econometrica 74(6), 1699-1714.

Vogelsang, T. (1998). Trend function hypothesis testing in the presence of serial correlation. Econometrica 66, 123-148. 


\section{A Appendices}

\section{A.1 Proofs of lemma 1}

Let $n_{d}=1$, from expression (4), we see that

$$
\begin{aligned}
T^{-1 / 2} \mathbf{x}_{[T r]} & =T^{-1 / 2} \mathbf{C} \sum_{i=1}^{[T r]} e^{\phi(T r-i) / T} \epsilon_{i}+\mathbf{C} \Psi\left[\begin{array}{c}
T^{-1} \sum_{i=1}^{[T r]} e^{\phi([T r]-i) / T} \\
T^{-2} \sum_{i=1}^{[T r]} i e^{\phi([T r]-i) / T}
\end{array}\right] \\
& +T^{-1 / 2} \mathbf{C}_{T}(L)\left(\epsilon_{[T r]}+\Psi \mathbf{B}_{T} \mathbf{d}_{[T r]}\right)+T^{-1 / 2} \mathbf{A}
\end{aligned}
$$

and $\left(T^{-1} \sum_{i=1}^{[T r]} e^{\phi([T r]-i) / T}, T^{-2} \sum_{i=1}^{[T r]} i e^{\phi([T r]-i) / T}\right) \underset{T \rightarrow \infty}{\rightarrow}\left(f_{\phi}(r), \int_{0}^{r} f_{\phi}(u) d u\right)$. Hence $\mathrm{E}\left[\mathbf{x}_{T}\right]=O\left(T^{1 / 2}\right)$ and $\mathbf{C} \sum_{i=1}^{t} e^{\phi(t-i) / T} \epsilon_{i}$ defines a - near if $\phi \neq 0-\mathbf{I}(1)$ stochastic trend so that $\mathbf{x}_{T} \sim O_{p}\left(T^{1 / 2}\right)$. Using expression (9), $T^{-1 / 2} \mathbf{x}_{[T r]} \Rightarrow \mathbf{C K}_{\boldsymbol{\Psi}, \phi}(r)$. This holds also for $n_{d}=0$ with corresponding adjustments to the definitions.

\section{A.2 Proof of propositions 2 and 3}

We follow the proof of Johansen (1996), starting with lemma 10.1 (denoted lemma J-10.1, and we use similar notation in the following). Since $\Pi_{T} \rightarrow \alpha \beta^{\prime}$ as $T \rightarrow \infty$, the expressions in lemma J-10.1 also hold here when considering $(t, T) \rightarrow(\infty, \infty)$. Hence, the empirical sums $S_{i j}$, for $i, j=0,1$, provide consistent estimates of the asymptotic conditional second moment, even though the residuals $R_{i j}$ are not corrected for the deterministic terms. Indeed, as $\mathbf{\Psi} \mathbf{B}_{T} \mathbf{d}_{t}=O_{p}\left(T^{-1 / 2}\right)$, we get:

$$
T^{-1} \sum_{t=1}^{T} \boldsymbol{\Psi} \mathbf{B}_{T} \mathbf{d}_{t} \mathbf{d}_{t}^{\prime} \mathbf{B}_{T}^{\prime} \Psi^{\prime}=\Psi\left[\begin{array}{cc}
\sum_{t=1}^{T} \frac{1}{T^{2}} & 0 \\
0 & \sum_{t=1}^{T} \frac{t^{2}}{T^{4}}
\end{array}\right] \boldsymbol{\Psi}_{T \rightarrow \infty}^{\prime} 0 .
$$

so that, from theorem J-14.1, with $\alpha_{1}=\mathbf{I}_{p}$ and $\beta_{1}=\left(e^{\phi / T}-1\right) \mathbf{I}_{p}$, under the conditions of a local-to-unit root and of a local deterministic trend, the following convergence results apply to the process generated by (6): $S_{00} \stackrel{P}{\rightarrow} \Sigma_{00}, \beta^{\prime} S_{11} \beta \stackrel{P}{\rightarrow}$ $\Sigma_{\beta \beta}$, and $\beta^{\prime} S_{10} \stackrel{P}{\rightarrow} \Sigma_{\beta 0}$. 
The notation we use is $\tau=\mathbf{C} \boldsymbol{\Psi} \mathbf{1}_{n_{d}+1}$, where $\mathbf{1}_{n_{d}+1}$ is a vector of ones of dimension $n_{d}+1$, and $\gamma$ orthogonal to $\beta$ and $\tau$ such that $(\beta, \tau, \gamma)$ has full rank $p$; also $\mathbf{f}_{\phi}(r)=$ $\left(f_{\phi}(r), \int_{0}^{r} f_{\phi}\right)^{\prime}$ if $n_{d}=1$ and $\mathbf{f}_{\phi}=f_{\phi}$ if $n_{d}=0$.

We assume $\tau \neq \mathbf{0}$. Therefore, as $T \rightarrow \infty$ and $r \in[0,1]$, define $\mathbf{G}(r)$ such that

$$
T^{-1 / 2}(\bar{\gamma}, \bar{\tau})^{\prime} \mathbf{x}_{[T r]} \Rightarrow \mathbf{G}(r)
$$

with

$$
\mathbf{G}(r)=\left[\begin{array}{c}
\bar{\gamma}^{\prime} \mathbf{C J}_{\phi}(r) \\
\bar{\tau}^{\prime} \mathbf{C K}_{\boldsymbol{\Psi}, \phi}(r)
\end{array}\right]
$$

When $n_{d}=0, \bar{\tau}^{\prime} \mathbf{C K}_{\boldsymbol{\Psi}, \phi}(r)=\bar{\tau}^{\prime} \mathbf{C J}_{\phi}(r)+f_{\phi}(r)$.

The case $n_{d}=0$ follows from theorems J-11.1 and J-14.4 with the results and notation above. These theorems also apply to our setting when appropriately replacing lemmata J-10.2, J-10.3 and J-14.3 with those given by our framework. This is what we do below and the proof of proposition 2 is then similar to those of theorems J-11.1 and J-14.4; we will not repeat them entirely here.

Since $\beta^{\prime} \alpha$ is invertible, $\beta_{\perp}\left(\alpha_{\perp}^{\prime} \beta_{\perp}\right)^{-1} \alpha_{\perp}^{\prime}+\alpha\left(\beta^{\prime} \alpha\right)^{-1} \beta^{\prime}=\mathbf{I}_{p}$ hence $\left(\beta, \alpha_{\perp}\right)$ has full rank. Moreover, as $\mathbf{C}=\beta_{\perp}\left(\alpha_{\perp}^{\prime} \beta_{\perp}\right)^{-1} \alpha_{\perp}^{\prime}$ and $\alpha$ and $\beta$ are defined via the space they span and their product, hence we can choose $\alpha_{\perp}=(\gamma, \tau)$. Then

$$
\mathbf{G}(r)=\alpha_{\perp}^{\prime} \mathbf{C J}_{\phi}(r)+\left(\mathbf{0}_{1 \times p-q-1}, \bar{\tau}^{\prime} \mathbf{C} \boldsymbol{\Psi} \mathbf{f}_{\phi}(r)\right)^{\prime}
$$

Equation (13) replaces lemma J-10.2, with

$$
\begin{aligned}
T^{-1}(\bar{\gamma}, \bar{\tau})^{\prime} S_{11}(\bar{\gamma}, \bar{\tau}) & \Rightarrow \int_{0}^{1} \mathbf{G G}^{\prime} d r \\
(\bar{\gamma}, \bar{\tau})^{\prime} S_{11} \beta & =O_{p}(1)
\end{aligned}
$$


and $\left\{S_{01}-\left(\alpha \beta^{\prime}+\left(e^{\phi / T}-1\right) \mathbf{I}_{p}\right) S_{11}\right\}(\bar{\gamma}, \bar{\tau})=T^{-1} \sum_{t} \mathbf{\Psi} \mathbf{B}_{T} \mathbf{d}_{t} R_{1}^{\prime}(\bar{\gamma}, \bar{\tau})+S_{\epsilon 1}(\bar{\gamma}, \bar{\tau})$ which weakly converges to

$$
\boldsymbol{\Psi} \int_{0}^{1}(1, r) \mathbf{G}^{\prime} d r+\Sigma \int_{0}^{1}(d \mathbf{W}) \mathbf{G}^{\prime}
$$

because $T^{1 / 2} \mathbf{B}_{T} \mathbf{d}_{[T r]} \rightarrow(1, r)$.

Equations (15) to (17) replace lemmata J-10.3 and J-14.3 in the proof. Define $S(\lambda)=\lambda S_{11}-S_{10} S_{00}^{-1} S_{01}$. Then, using $T^{-1 / 2}(\bar{\gamma}, \bar{\tau})^{\prime} \mathbf{x}_{[T r]} \Rightarrow \mathbf{G}(r)$, as $(\beta, \tau, \gamma)$ is of full rank:

$$
\begin{aligned}
& \left|\left(\beta, T^{-1 / 2} \bar{\gamma}, T^{-1 / 2} \bar{\tau}\right)^{\prime} S(\lambda)\left(\beta, T^{-1 / 2} \bar{\gamma}, T^{-1 / 2} \bar{\tau}\right)\right| \\
& \Rightarrow\left|\left[\begin{array}{cc}
\lambda \Sigma_{\beta \beta} & 0 \\
0 & \lambda\left(\int_{0}^{1} \mathbf{G G}^{\prime} d r\right)
\end{array}\right]-\left[\begin{array}{cc}
\Sigma_{\beta 0} \Sigma_{00}^{-1} \Sigma_{0 \beta} & 0 \\
0 & 0
\end{array}\right]\right| \\
& =\left|\lambda \Sigma_{\beta \beta}-\Sigma_{\beta 0} \Sigma_{00}^{-1} \Sigma_{0 \beta}\right|\left|\lambda\left(\int_{0}^{1} \mathbf{G G}^{\prime} d r\right)\right|
\end{aligned}
$$

which has $p-q$ zero roots and $q$ positive roots. We can also write

$$
\begin{aligned}
& \left|\left(\beta, T^{-1 / 2} \bar{\gamma}, T^{-1 / 2} \bar{\tau}\right)^{\prime} S(\lambda)\left(\beta, T^{-1 / 2} \bar{\gamma}, T^{-1 / 2} \bar{\tau}\right)\right| \\
& =\left|\beta^{\prime} S(\lambda) \beta\right| \mid\left[\begin{array}{c}
T^{-1 / 2} \bar{\gamma}^{\prime} \\
T^{-1 / 2} \bar{\tau}^{\prime}
\end{array}\right]\left\{S(\lambda)-S(\lambda) \beta\left[\beta^{\prime} S(\lambda) \beta\right]^{-1} \beta^{\prime} S(\lambda)\right\}\left[\begin{array}{c}
T^{-1 / 2} \bar{\gamma}^{\prime} \\
T^{-1 / 2} \bar{\tau}^{\prime}
\end{array}\right]^{\prime}
\end{aligned}
$$

Now, let $\rho=\lambda T$ fixed and $(\lambda, T) \rightarrow(0, \infty)$. We show that the $p-q$ smallest root of $S(\lambda)$ decrease to zero at the rate $T^{-1}$ and that $T \widehat{\lambda}$ converges:

$$
\beta^{\prime} S(\lambda) \beta=\rho T^{-1} \beta^{\prime} S_{11} \beta-\beta^{\prime} S_{10} S_{00}^{-1} S_{01} \beta \stackrel{P}{\rightarrow}-\Sigma_{\beta 0} \Sigma_{00}^{-1} \Sigma_{0 \beta}
$$

so that $\left|\beta^{\prime} S(\lambda) \beta\right|$ has no roots. Additionally

$$
T^{-1 / 2}(\bar{\gamma}, \bar{\tau})^{\prime} S(\lambda) \beta=-T^{-1 / 2}(\bar{\gamma}, \bar{\tau})^{\prime} S_{10} \Sigma_{00}^{-1} \Sigma_{0 \beta}+o_{p}(1)
$$

and

$$
\begin{aligned}
& (\bar{\gamma}, \bar{\tau})^{\prime}\left\{S(\lambda)-S(\lambda) \beta\left[\beta^{\prime} S(\lambda) \beta\right]^{-1} \beta^{\prime} S(\lambda)\right\}(\bar{\gamma}, \bar{\tau}) \\
& =\rho T^{-1}(\bar{\gamma}, \bar{\tau})^{\prime} S_{11}(\bar{\gamma}, \bar{\tau})-(\bar{\gamma}, \bar{\tau})^{\prime} S_{10} \Lambda S_{01}(\bar{\gamma}, \bar{\tau})+o_{p}(1)
\end{aligned}
$$


with

$$
\boldsymbol{\Lambda}=\Sigma_{00}^{-1}-\Sigma_{00}^{-1} \Sigma_{0 \beta}\left[\Sigma_{\beta 0} \Sigma_{00}^{-1} \Sigma_{0 \beta}\right]^{-1} \Sigma_{\beta 0} \Sigma_{00}^{-1}=\alpha_{\perp}\left(\alpha_{\perp}^{\prime} \Omega \alpha_{\perp}\right)^{-1} \alpha_{\perp}^{\prime}
$$

From (13), $\mathbf{G}$ is the projection of $\mathbf{C K}_{\Psi, \phi}$ on $(\gamma, \tau)=\alpha_{\perp}$, hence

$$
(\bar{\gamma}, \bar{\tau})^{\prime} S_{10} \alpha_{\perp}=(\bar{\gamma}, \bar{\tau})^{\prime} S_{10}(\bar{\gamma}, \bar{\tau}) \Rightarrow \int_{0}^{1} \mathbf{G}(d \mathbf{G})^{\prime}
$$

Combining (15), (19) and (20), we see that, according to (18), the roots $\rho$ asymptotically satisfy

$$
\left|\rho\left(\int_{0}^{1} \mathbf{G G}^{\prime} d r\right)-\left(\int_{0}^{1} \mathbf{G} d \mathbf{G}^{\prime}\right)\left(\alpha_{\perp}^{\prime} \mathbf{\Omega} \alpha_{\perp}\right)^{-1}\left(\int_{0}^{1} \mathbf{G} d \mathbf{G}^{\prime}\right)^{\prime}\right|=0 .
$$

Hence given that the roots of $(21)|S(\lambda)|$ tend to zero at rate $O_{p}\left(T^{-1}\right)$. Rescale the diffusion $\mathbf{G}$ as $\mathbf{G}_{0}=\left(\alpha_{\perp}^{\prime} \mathbf{C} \Omega \mathbf{C}^{\prime} \alpha_{\perp}\right)^{-1 / 2} \mathbf{G}$ to obtain the result: the likelihood ratio statistic $T \sum_{i=q+1}^{p} \log \left(1-\widehat{\lambda}_{i}\right)$ satisfies

$$
\begin{aligned}
& -2 \log Q(H(q) \mid H(p)) \\
& \Rightarrow \operatorname{tr}\left\{\int_{0}^{1} d \mathbf{G}_{0} \mathbf{G}_{0}^{\prime}\left[\int_{0}^{1} \mathbf{G}_{0} \mathbf{G}_{0}^{\prime}\right]^{-1} \int_{0}^{1} \mathbf{G}_{0}\left(d \mathbf{G}_{0}\right)^{\prime}\right\}
\end{aligned}
$$

In the presence of a deterministic component entered unrestrictedly in the regression model (so that it is partialled out as the lagged differences in $Z_{2}$ ), $\mathbf{G}_{0}$ in (22) is then corrected for a mean $\left(n_{d}=0\right)$, or both a mean and a linear trend $\left(n_{d}=1\right)$. Given that $\mathbf{f}_{\phi}(r)$ is nonlinear, the regression coefficients will be nonzero for $\phi \neq 0$.

Should the modeler suspect the presence of a deterministic trend in the process, following Johansen (1994), she would test for the null hypothesis $H^{*}(q): \Pi=\alpha \beta^{\prime}$ and $\boldsymbol{\Psi}_{\mathrm{t}}=\boldsymbol{\Psi}_{0}+\boldsymbol{\Psi}_{1} t$ with $\alpha_{\perp}^{\prime} \boldsymbol{\Psi}_{1}=\mathbf{0}$; this leads to setting the model with $\mathbf{X}_{t-1}=$ $\left(\mathbf{x}_{t-1}, t\right)$ instead of $\mathbf{x}_{t-1}$ entering the cointegrating space. Then $\tau=\mathbf{C} \boldsymbol{\Psi}_{0} \mathbf{1}_{n_{d}+1}$ and G only depends on $\boldsymbol{\Psi}$ via $\boldsymbol{\Psi}_{0}$. Now the LR statistic admits a different asymptotic 
distribution:

$$
\begin{aligned}
& -2 \log Q\left(H^{*}(q) \mid H^{*}(p)\right) \\
& \Rightarrow \operatorname{tr}\left\{\int_{0}^{1}\left(d \mathbf{G}_{0}\right) \overline{\mathbf{G}}^{\prime}\left[\int_{0}^{1} \overline{\mathbf{G G}^{\prime}}\right]^{-1} \int_{0}^{1} \overline{\mathbf{G}}\left(d \mathbf{G}_{0}\right)^{\prime}\right\} .
\end{aligned}
$$

where $\overline{\mathbf{G}}=\left(\mathbf{G}_{0}, r\right)$ corrected for the mean and it is function of $\mathbf{\Psi}_{0}$. 


\begin{tabular}{|c|c|c|c|c|c|c|}
\hline & \multirow{2}{*}{$\begin{array}{c}\text { Number of } \\
\text { stochastic trends }\end{array}$} & \multicolumn{5}{|c|}{ Probability that $\mathrm{LR}_{\mu}$ is less than entry } \\
\hline & & 0.5 & 0.9 & 0.95 & 0.975 & 0.99 \\
\hline \multicolumn{7}{|c|}{ Asymptotic $^{a}$} \\
\hline & 1 & 2.42 & 6.69 & 8.08 & 9.66 & 11.58 \\
\hline & 2 & 9.34 & 15.58 & 17.84 & 19.61 & 21.96 \\
\hline & 3 & 20.19 & 28.44 & 31.26 & 34.06 & 37.29 \\
\hline & 4 & 34.87 & 45.25 & 48.42 & 51.80 & 55.55 \\
\hline & 5 & 55.37 & 65.96 & 69.98 & 73.03 & 77.91 \\
\hline \multicolumn{7}{|c|}{ Ahn-Reinsel $^{b}$ for $T=25$} \\
\hline & 1 & 2.52 & 6.97 & 8.42 & 10.06 & 12.06 \\
\hline & 2 & 10.15 & 16.94 & 19.40 & 21.32 & 23.87 \\
\hline & 3 & 22.94 & 32.31 & 35.52 & 38.71 & 42.38 \\
\hline & 4 & 41.52 & 53.87 & 57.64 & 61.67 & 66.13 \\
\hline & 5 & 69.22 & 82.45 & 87.47 & 91.29 & 97.39 \\
\hline \multicolumn{7}{|c|}{ Finite Sample, simulated with $T=25$} \\
\hline \multirow[t]{5}{*}{$\psi=0$} & 1 & 2.13 & 5.43 & 6.66 & 7.85 & 9.51 \\
\hline & 2 & 8.47 & 14.09 & 16.07 & 18.06 & 20.17 \\
\hline & 3 & 18.99 & 27.23 & 29.99 & 32.62 & 35.49 \\
\hline & 4 & 34.18 & 44.80 & 48.42 & 51.76 & 55.62 \\
\hline & 5 & 54.49 & 68.36 & 72.98 & 77.37 & 82.40 \\
\hline \multirow[t]{5}{*}{$\psi=0.25$} & 1 & 2.12 & 5.37 & 6.56 & 7.68 & 9.36 \\
\hline & 2 & 8.37 & 13.97 & 16.05 & 17.86 & 20.24 \\
\hline & 3 & 18.92 & 27.14 & 29.93 & 32.34 & 35.50 \\
\hline & 4 & 34.09 & 44.83 & 48.42 & 51.60 & 56.02 \\
\hline & 5 & 54.42 & 68.33 & 73.03 & 77.55 & 82.58 \\
\hline \multirow[t]{5}{*}{$\psi=0.5$} & 1 & 2.01 & 5.26 & 6.51 & 7.67 & 9.25 \\
\hline & 2 & 8.23 & 13.82 & 15.85 & 17.68 & 20.00 \\
\hline & 3 & 18.72 & 26.87 & 29.59 & 32.23 & 35.36 \\
\hline & 4 & 33.88 & 44.57 & 48.12 & 51.55 & 55.86 \\
\hline & 5 & 54.19 & 68.14 & 72.66 & 77.09 & 82.76 \\
\hline \multirow[t]{5}{*}{$\psi=1$} & 1 & 1.57 & 4.90 & 6.08 & 7.27 & 8.91 \\
\hline & 2 & 7.74 & 13.21 & 15.19 & 16.99 & 19.53 \\
\hline & 3 & 18.20 & 26.20 & 28.93 & 31.38 & 34.74 \\
\hline & 4 & 33.43 & 44.00 & 47.62 & 50.86 & 55.00 \\
\hline & 5 & 53.70 & 67.70 & 72.05 & 76.12 & 81.98 \\
\hline \multirow[t]{5}{*}{$\psi=2$} & 1 & 0.77 & 3.67 & 4.77 & 5.82 & 7.23 \\
\hline & 2 & 7.10 & 12.27 & 14.04 & 15.74 & 18.05 \\
\hline & 3 & 17.65 & 25.50 & 28.06 & 30.51 & 33.65 \\
\hline & 4 & 33.03 & 43.50 & 47.13 & 50.62 & 54.71 \\
\hline & 5 & 53.50 & 67.32 & 71.84 & 75.59 & 80.59 \\
\hline
\end{tabular}

Table 1: Comparison of the distributions of the Likelihood Ratio test where a constant enters unrestrictedly in the model. The first five rows report the asymptotic quantiles recorded in the appendix of Hamilton (1994). The following rows report respectively the finite sample approximation of Ann-Reinsel over samples of $T=25$ observations. The remaining rows record simulated finite sample distributions under the null and local alternatives when the data generating process presents a drift lying in the space spanned by the common stoghastic trends. The drift is parameterized as $T^{-1 / 2}(\psi, 0, \ldots)^{\prime}$. 
Table 2: The table reports the Monte Carlo $j$ quantiles $x_{j}$ of the distribution of the LR statistics for model $\mathrm{M}_{0}$ under the null of $p-q$ common stochastic trends and in the presence of a local deterministic trend with parameter $\psi$. The table also reports the $p$ values of the Mann-Whitney test statistics for the test of identical distributions between the finite sample distribution of the LR statistic and its local-asymptotic approximation under the local alternative of near-stochastic trends parameterized by $\phi$. The number of Monte Carlo replications is 20,000 and the sample size $T$.

\begin{tabular}{|c|c|c|c|c|c|c|c|c|c|c|c|c|c|}
\hline \multirow[b]{2}{*}{$T$} & \multirow[b]{2}{*}{$\psi$} & \multirow[b]{2}{*}{$p-q$} & \multirow[b]{2}{*}{$x_{50}$} & \multirow[b]{2}{*}{$x_{90}$} & \multirow[b]{2}{*}{$x_{95}$} & \multirow[b]{2}{*}{$x_{97.5}$} & \multirow[b]{2}{*}{$x_{99}$} & \multicolumn{6}{|c|}{$p$-values of the Mann-Whitney test statistic in \% } \\
\hline & & & & & & & & $\phi=1$ & $\phi=.75$ & $\phi=.5$ & $\phi=.25$ & $\phi=0$ & $\phi=-0.25$ \\
\hline 50 & 0 & 1 & 0.59 & 2.96 & 4.13 & 5.34 & 6.78 & 0.0 & 0.0 & 0.0 & 35.2 & 57.7 & 15.1 \\
\hline 50 & 0 & 2 & 5.56 & 10.50 & 12.34 & 14.14 & 16.20 & 0.0 & 0.0 & 0.0 & 0.1 & 44.5 & 0.0 \\
\hline 50 & 0 & 3 & 14.70 & 22.39 & 24.77 & 27.37 & 30.06 & 0.0 & 0.0 & 0.0 & 0.8 & 0.1 & 0.0 \\
\hline 50 & 0 & 4 & 28.61 & 38.30 & 41.43 & 44.31 & 48.04 & 0.0 & 0.0 & 0.0 & 4.0 & 0.0 & 0.0 \\
\hline 50 & 0 & 5 & 47.00 & 59.59 & 63.71 & 67.63 & 72.68 & 0.0 & 0.0 & 29.3 & 0.0 & 0.0 & 0.0 \\
\hline 50 & 0.25 & 1 & 0.61 & 3.10 & 4.25 & 5.45 & 7.05 & 0.0 & 0.0 & 0.0 & 1.6 & 96.0 & 17.6 \\
\hline 50 & 0.25 & 2 & 5.59 & 10.69 & 12.45 & 14.05 & 16.44 & 0.0 & 0.0 & 0.0 & 0.0 & 0.9 & 0.0 \\
\hline 50 & 0.25 & 3 & 14.84 & 22.25 & 24.77 & 27.05 & 29.67 & 0.0 & 0.0 & 0.0 & 11.0 & 1.3 & 0.0 \\
\hline 50 & 0.25 & 4 & 28.54 & 38.32 & 41.39 & 44.00 & 47.87 & 0.0 & 0.0 & 0.0 & 9.5 & 0.0 & 0.0 \\
\hline 50 & 0.25 & 5 & 46.88 & 59.34 & 63.71 & 67.41 & 72.40 & 0.0 & 0.0 & 20.2 & 0.0 & 0.0 & 0.0 \\
\hline 50 & 0.5 & 1 & 0.63 & 3.04 & 4.32 & 5.51 & 7.12 & 0.0 & 0.0 & 0.0 & 1.7 & 32.0 & 86.1 \\
\hline 50 & 0.5 & 2 & 5.68 & 10.80 & 12.61 & 14.57 & 16.67 & 0.0 & 0.0 & 0.0 & 0.0 & 55.7 & 1.0 \\
\hline 50 & 0.5 & 3 & 14.90 & 22.65 & 25.30 & 27.81 & 30.53 & 0.0 & 0.0 & 0.0 & 0.0 & 77.3 & 0.0 \\
\hline 50 & 0.5 & 4 & 28.65 & 38.60 & 42.01 & 44.83 & 48.69 & 0.0 & 0.0 & 0.0 & 1.1 & 0.0 & 0.0 \\
\hline 50 & 0.5 & 5 & 47.23 & 59.86 & 64.00 & 67.88 & 72.04 & 0.0 & 0.0 & 0.0 & 3.4 & 0.0 & 0.0 \\
\hline 50 & 1 & 1 & 0.69 & 3.56 & 4.99 & 6.29 & 7.87 & 0.0 & 0.0 & 0.0 & 0.0 & 39.8 & 0.0 \\
\hline 50 & 1 & 2 & 5.94 & 11.31 & 13.02 & 14.82 & 16.87 & 0.0 & 0.0 & 0.0 & 0.0 & 0.0 & 0.3 \\
\hline 50 & 1 & 3 & 15.36 & 22.97 & 25.45 & 27.74 & 30.76 & 0.0 & 0.0 & 0.0 & 0.0 & 0.0 & 82.5 \\
\hline 50 & 1 & 4 & 29.09 & 39.47 & 42.84 & 45.97 & 49.45 & 0.0 & 0.0 & 0.0 & 0.0 & 0.0 & 79.7 \\
\hline 50 & 1 & 5 & 47.64 & 60.23 & 64.26 & 67.99 & 72.78 & 0.0 & 0.0 & 0.0 & 0.0 & 0.0 & 0.0 \\
\hline 100 & 0 & 1 & 0.61 & 2.88 & 4.02 & 5.20 & 6.72 & 0.0 & 0.0 & 0.0 & 6.2 & 42.9 & 61.7 \\
\hline 100 & 0 & 2 & 5.53 & 10.53 & 12.37 & 14.13 & 16.37 & 0.0 & 0.0 & 0.0 & 1.1 & 21.9 & 1.4 \\
\hline 100 & 0 & 3 & 14.66 & 21.97 & 24.54 & 27.25 & 30.10 & 0.0 & 0.0 & 0.0 & 0.9 & 5.8 & 0.0 \\
\hline 100 & 0 & 4 & 27.95 & 37.72 & 41.01 & 43.99 & 47.70 & 0.0 & 0.0 & 0.0 & 4.8 & 2.0 & 0.0 \\
\hline 100 & 0 & 5 & 45.64 & 58.05 & 62.14 & 65.75 & 70.38 & 0.0 & 0.0 & 0.0 & 16.9 & 0.0 & 0.0 \\
\hline 100 & 0.25 & 1 & 0.60 & 2.91 & 4.00 & 5.21 & 6.75 & 0.0 & 0.0 & 0.0 & 8.7 & 96.5 & 97.1 \\
\hline 100 & 0.25 & 2 & 5.58 & 10.73 & 12.61 & 14.31 & 16.42 & 0.0 & 0.0 & 0.0 & 0.0 & 57.5 & 0.0 \\
\hline 100 & 0.25 & 3 & 14.75 & 22.20 & 24.75 & 27.32 & 30.22 & 0.0 & 0.0 & 0.0 & 0.0 & 0.8 & 0.0 \\
\hline 100 & 0.25 & 4 & 28.05 & 37.75 & 40.94 & 43.63 & 47.46 & 0.0 & 0.0 & 0.0 & 0.0 & 0.0 & 0.0 \\
\hline 100 & 0.25 & 5 & 45.64 & 57.81 & 61.75 & 65.05 & 70.25 & 0.0 & 0.0 & 0.0 & 1.1 & 0.0 & 0.0 \\
\hline 100 & 0.5 & 1 & 0.60 & 2.99 & 4.21 & 5.51 & 7.28 & 0.0 & 0.0 & 0.0 & 2.0 & 60.0 & 40.9 \\
\hline 100 & 0.5 & 2 & 5.67 & 10.89 & 12.71 & 14.34 & 16.69 & 0.0 & 0.0 & 0.0 & 0.0 & 2.5 & 4.7 \\
\hline 100 & 0.5 & 3 & 14.63 & 22.25 & 24.88 & 27.25 & 29.99 & 0.0 & 0.0 & 0.0 & 0.0 & 28.8 & 0.0 \\
\hline 100 & 0.5 & 4 & 28.14 & 38.03 & 41.38 & 44.13 & 47.31 & 0.0 & 0.0 & 0.0 & 0.0 & 39.3 & 0.0 \\
\hline 100 & 0.5 & 5 & 45.83 & 58.01 & 61.88 & 65.67 & 69.51 & 0.0 & 0.0 & 0.0 & 0.0 & 2.4 & 0.0 \\
\hline 100 & 1 & 1 & 0.69 & 3.54 & 4.92 & 6.10 & 8.15 & 0.0 & 0.0 & 0.0 & 0.0 & 95.7 & 0.3 \\
\hline 100 & 1 & 2 & 5.96 & 11.48 & 13.55 & 15.44 & 17.82 & 0.0 & 0.0 & 0.0 & 0.0 & 0.0 & 90.8 \\
\hline 100 & 1 & 3 & 15.14 & 23.07 & 25.57 & 27.81 & 30.74 & 0.0 & 0.0 & 0.0 & 0.0 & 0.0 & 1.4 \\
\hline 100 & 1 & 4 & 28.64 & 38.58 & 41.76 & 44.55 & 48.60 & 0.0 & 0.0 & 0.0 & 0.0 & 0.0 & 0.5 \\
\hline 100 & 1 & 5 & 46.21 & 58.61 & 62.30 & 65.37 & 70.35 & 0.0 & 0.0 & 0.0 & 0.0 & 0.0 & 31.4 \\
\hline 200 & 0 & 1 & 0.62 & 2.96 & 4.22 & 5.41 & 7.13 & 0.0 & 0.0 & 0.0 & 2.6 & 27.0 & 41.3 \\
\hline 200 & 0 & 2 & 5.52 & 10.59 & 12.45 & 14.33 & 16.56 & 0.0 & 0.0 & 0.0 & 0.4 & 42.9 & 4.0 \\
\hline 200 & 0 & 3 & 14.51 & 21.77 & 24.32 & 26.78 & 29.78 & 0.0 & 0.0 & 0.0 & 3.2 & 37.7 & 0.0 \\
\hline 200 & 0 & 4 & 27.72 & 37.45 & 40.72 & 43.55 & 46.67 & 0.0 & 0.0 & 0.0 & 0.0 & 16.2 & 0.0 \\
\hline 200 & 0 & 5 & 45.03 & 57.24 & 60.87 & 64.13 & 68.97 & 0.0 & 0.0 & 0.0 & 0.0 & 0.0 & 0.0 \\
\hline 200 & 0.25 & 1 & 0.61 & 2.96 & 4.15 & 5.45 & 7.20 & 0.0 & 0.0 & 0.0 & 5.3 & 63.3 & 49.1 \\
\hline 200 & 0.25 & 2 & 5.56 & 10.50 & 12.34 & 13.92 & 16.04 & 0.0 & 0.0 & 0.0 & 0.0 & 7.3 & 0.1 \\
\hline 200 & 0.25 & 3 & 14.51 & 21.92 & 24.72 & 27.10 & 30.40 & 0.0 & 0.0 & 0.0 & 0.0 & 42.2 & 0.2 \\
\hline 200 & 0.25 & 4 & 27.72 & 37.25 & 40.33 & 43.25 & 46.83 & 0.0 & 0.0 & 0.0 & 0.0 & 70.6 & 0.0 \\
\hline 200 & 0.25 & 5 & 45.08 & 57.17 & 60.83 & 64.31 & 68.61 & 0.0 & 0.0 & 0.0 & 0.1 & 0.2 & 0.0 \\
\hline 200 & 0.5 & 1 & 0.60 & 3.16 & 4.32 & 5.45 & 7.13 & 0.0 & 0.0 & 0.0 & 1.1 & 44.4 & 75.3 \\
\hline 200 & 0.5 & 2 & 5.66 & 10.80 & 12.67 & 14.47 & 16.84 & 0.0 & 0.0 & 0.0 & 0.0 & 45.7 & 18.5 \\
\hline 200 & 0.5 & 3 & 14.56 & 22.22 & 24.78 & 27.19 & 29.96 & 0.0 & 0.0 & 0.0 & 0.0 & 63.7 & 0.0 \\
\hline 200 & 0.5 & 4 & 27.93 & 37.54 & 40.50 & 43.59 & 47.25 & 0.0 & 0.0 & 0.0 & 0.0 & 9.5 & 0.0 \\
\hline 200 & 0.5 & 5 & 45.07 & 57.34 & 61.31 & 64.58 & 68.58 & 0.0 & 0.0 & 0.0 & 0.0 & 79.7 & 0.0 \\
\hline 200 & 1 & 1 & 0.70 & 3.66 & 5.07 & 6.40 & 8.52 & 0.0 & 0.0 & 0.0 & 0.0 & 48.7 & 0.0 \\
\hline 200 & 1 & 2 & 5.88 & 11.37 & 13.38 & 15.43 & 17.58 & 0.0 & 0.0 & 0.0 & 0.0 & 0.0 & 62.6 \\
\hline 200 & 1 & 3 & 15.07 & 22.73 & 25.23 & 27.75 & 31.07 & 0.0 & 0.0 & 0.0 & 0.0 & 0.0 & 0.1 \\
\hline 200 & 1 & 4 & 28.33 & 38.21 & 41.64 & 44.21 & 47.71 & 0.0 & 0.0 & 0.0 & 0.0 & 0.0 & 0.1 \\
\hline 200 & 1 & 5 & 45.60 & 57.64 & 61.37 & 65.12 & 69.52 & 0.0 & 0.0 & 0.0 & 0.0 & 0.0 & 0.1 \\
\hline
\end{tabular}


Table 3: The table reports the Monte Carlo $j$ quantiles $x_{j}$ of the distribution of the LR statistics for model $\mathrm{M}_{\mu}$ under the null of $p-q$ common stochastic trends and in the presence of a local deterministic trend with parameter $\psi$. The table also reports the $p$ values of the Mann-Whitney test statistics for the test of identical distributions between the finite sample distribution of the LR statistic and its local-asymptotic approximation under the local alternative of near-stochastic trends parameterized by $\phi$. The number of Monte Carlo replications is 20,000 and the sample size $T$.

\begin{tabular}{|c|c|c|c|c|c|c|c|c|c|c|c|c|c|}
\hline \multirow[b]{2}{*}{$T$} & \multirow[b]{2}{*}{$\psi$} & \multirow[b]{2}{*}{$p-q$} & \multirow[b]{2}{*}{$x_{50}$} & \multirow[b]{2}{*}{$x_{90}$} & \multirow[b]{2}{*}{$x_{95}$} & \multirow[b]{2}{*}{$x_{97.5}$} & \multirow[b]{2}{*}{$x_{99}$} & \multicolumn{6}{|c|}{$p$-values of the Mann-Whitney test statistic in \% } \\
\hline & & & & & & & & $\phi=1$ & $\phi=.75$ & $\phi=.5$ & $\phi=.25$ & $\phi=0$ & $\phi=-0.25$ \\
\hline 50 & 0 & 1 & 2.25 & 5.81 & 7.25 & 8.52 & 9.92 & 0.0 & 0.0 & 0.0 & 15.0 & 0.0 & 0.0 \\
\hline 50 & 0 & 2 & 8.88 & 14.51 & 16.45 & 18.38 & 20.78 & 0.0 & 0.1 & 30.0 & 0.0 & 0.0 & 0.0 \\
\hline 50 & 0 & 3 & 19.42 & 27.29 & 29.89 & 32.34 & 35.59 & 0.0 & 60.0 & 7.3 & 0.0 & 0.0 & 0.0 \\
\hline 50 & 0 & 4 & 34.41 & 44.33 & 47.76 & 50.62 & 54.27 & 1.3 & 79.7 & 11.1 & 0.0 & 0.0 & 0.0 \\
\hline 50 & 0 & 5 & 53.91 & 66.45 & 70.37 & 74.07 & 78.80 & 0.0 & 4.2 & 31.8 & 39.3 & 0.0 & 2.8 \\
\hline 50 & 0.25 & 1 & 2.22 & 6.04 & 7.40 & 8.62 & 10.47 & 0.0 & 0.0 & 0.0 & 78.7 & 0.0 & 0.0 \\
\hline 50 & 0.25 & 2 & 8.85 & 14.71 & 16.69 & 18.42 & 20.82 & 0.0 & 0.0 & 65.7 & 0.0 & 0.0 & 0.0 \\
\hline 50 & 0.25 & 3 & 19.47 & 27.32 & 29.61 & 32.11 & 34.40 & 10.9 & 98.9 & 0.8 & 0.0 & 0.0 & 0.0 \\
\hline 50 & 0.25 & 4 & 34.28 & 44.50 & 47.78 & 50.79 & 54.55 & 34.6 & 67.3 & 3.3 & 0.0 & 0.0 & 0.0 \\
\hline 50 & 0.25 & 5 & 53.84 & 66.31 & 70.52 & 73.90 & 78.85 & 0.2 & 0.0 & 72.1 & 33.4 & 0.6 & 0.5 \\
\hline 50 & 0.5 & 1 & 2.14 & 5.78 & 7.08 & 8.37 & 9.89 & 0.0 & 0.0 & 0.0 & 20.0 & 0.0 & 0.0 \\
\hline 50 & 0.5 & 2 & 8.78 & 14.49 & 16.57 & 18.32 & 20.40 & 0.0 & 0.0 & 10.0 & 3.5 & 0.0 & 0.0 \\
\hline 50 & 0.5 & 3 & 19.46 & 27.42 & 29.94 & 32.34 & 35.68 & 23.1 & 65.2 & 1.2 & 0.0 & 0.0 & 0.0 \\
\hline 50 & 0.5 & 4 & 34.24 & 44.49 & 47.84 & 50.81 & 54.18 & 0.9 & 38.1 & 49.5 & 3.2 & 0.0 & 0.0 \\
\hline 50 & 0.5 & 5 & 53.83 & 66.34 & 70.49 & 74.16 & 79.22 & 7.5 & 0.2 & 4.7 & 5.7 & 0.1 & 0.0 \\
\hline 50 & 1 & 1 & 1.72 & 5.55 & 6.92 & 8.04 & 9.74 & 0.0 & 0.0 & 0.0 & 6.6 & 0.0 & 0.0 \\
\hline 50 & 1 & 2 & 8.51 & 14.05 & 15.89 & 17.63 & 19.71 & 0.0 & 0.0 & 0.0 & 41.5 & 0.0 & 0.0 \\
\hline 50 & 1 & 3 & 19.21 & 26.80 & 29.57 & 31.92 & 34.83 & 73.5 & 35.2 & 50.7 & 1.2 & 0.0 & 0.0 \\
\hline 50 & 1 & 4 & 34.13 & 44.45 & 47.57 & 50.91 & 54.66 & 14.2 & 7.9 & 90.2 & 0.1 & 0.0 & 0.0 \\
\hline 50 & 1 & 5 & 53.63 & 66.07 & 69.87 & 73.14 & 78.00 & 12.2 & 92.4 & 0.4 & 50.4 & 44.5 & 1.9 \\
\hline 100 & 0 & 1 & 2.37 & 6.31 & 7.74 & 9.06 & 10.66 & 0.0 & 0.0 & 0.0 & 20.0 & 27.4 & 0.0 \\
\hline 100 & 0 & 2 & 9.17 & 15.38 & 17.55 & 19.59 & 22.09 & 0.0 & 0.0 & 0.6 & 53.8 & 0.3 & 0.0 \\
\hline 100 & 0 & 3 & 19.88 & 28.16 & 30.80 & 33.33 & 36.65 & 0.0 & 0.1 & 11.4 & 10.8 & 0.0 & 0.0 \\
\hline 100 & 0 & 4 & 34.90 & 45.48 & 48.72 & 51.64 & 55.39 & 0.0 & 0.0 & 43.0 & 1.2 & 0.0 & 0.0 \\
\hline 100 & 0 & 5 & 54.09 & 66.83 & 71.05 & 74.71 & 79.13 & 0.0 & 0.0 & 2.1 & 93.1 & 16.4 & 6.7 \\
\hline 100 & 0.25 & 1 & 2.38 & 6.15 & 7.60 & 8.84 & 10.71 & 0.0 & 0.0 & 0.0 & 3.4 & 11.7 & 0.0 \\
\hline 100 & 0.25 & 2 & 9.19 & 15.29 & 17.38 & 19.32 & 21.70 & 0.0 & 0.0 & 0.1 & 32.6 & 0.2 & 0.4 \\
\hline 100 & 0.25 & 3 & 20.00 & 28.19 & 30.96 & 33.55 & 36.70 & 0.0 & 0.9 & 45.5 & 11.9 & 24.1 & 0.0 \\
\hline 100 & 0.25 & 4 & 34.96 & 45.26 & 48.45 & 51.10 & 55.28 & 0.0 & 0.9 & 48.1 & 2.0 & 0.9 & 0.3 \\
\hline 100 & 0.25 & 5 & 53.93 & 66.71 & 70.77 & 74.50 & 79.22 & 0.0 & 0.0 & 13.1 & 5.0 & 28.1 & 2.0 \\
\hline 100 & 0.5 & 1 & 2.22 & 6.05 & 7.61 & 8.98 & 10.83 & 0.0 & 0.0 & 0.0 & 0.0 & 17.5 & 0.0 \\
\hline 100 & 0.5 & 2 & 9.12 & 15.19 & 17.35 & 19.39 & 21.61 & 0.0 & 0.0 & 2.8 & 16.4 & 0.0 & 0.0 \\
\hline 100 & 0.5 & 3 & 19.83 & 28.05 & 30.48 & 33.10 & 35.94 & 0.0 & 0.0 & 0.0 & 8.2 & 52.9 & 0.0 \\
\hline 100 & 0.5 & 4 & 34.82 & 45.04 & 48.19 & 51.13 & 54.76 & 0.0 & 0.0 & 40.5 & 42.9 & 2.5 & 0.0 \\
\hline 100 & 0.5 & 5 & 54.03 & 66.60 & 70.34 & 73.75 & 78.64 & 0.0 & 0.1 & 0.8 & 68.7 & 6.2 & 11.3 \\
\hline 100 & 1 & 1 & 1.85 & 5.73 & 7.10 & 8.53 & 10.21 & 0.0 & 0.0 & 0.0 & 0.3 & 2.4 & 0.0 \\
\hline 100 & 1 & 2 & 8.83 & 14.85 & 17.09 & 18.99 & 21.26 & 0.0 & 0.0 & 0.0 & 0.0 & 67.9 & 1.9 \\
\hline 100 & 1 & 3 & 19.64 & 27.82 & 30.47 & 33.28 & 36.09 & 0.0 & 0.0 & 0.0 & 3.7 & 5.8 & 0.1 \\
\hline 100 & 1 & 4 & 34.72 & 44.73 & 47.90 & 50.95 & 55.34 & 23.9 & 0.8 & 0.6 & 46.4 & 4.7 & 17.9 \\
\hline 100 & 1 & 5 & 53.79 & 66.29 & 70.07 & 73.53 & 77.16 & 59.3 & 0.0 & 0.1 & 34.6 & 52.8 & 70.8 \\
\hline 200 & 0 & 1 & 2.48 & 6.45 & 8.00 & 9.50 & 11.24 & 0.0 & 0.0 & 0.0 & 0.2 & 20.8 & 0.1 \\
\hline 200 & 0 & 2 & 9.37 & 15.60 & 17.61 & 19.66 & 22.30 & 0.0 & 0.0 & 0.0 & 1.0 & 3.4 & 0.0 \\
\hline 200 & 0 & 3 & 20.22 & 28.70 & 31.48 & 34.02 & 37.30 & 0.0 & 0.0 & 2.9 & 10.0 & 10.3 & 0.3 \\
\hline 200 & 0 & 4 & 35.10 & 45.65 & 49.18 & 52.66 & 56.24 & 0.0 & 0.0 & 2.7 & 27.0 & 7.8 & 0.0 \\
\hline 200 & 0 & 5 & 54.35 & 66.92 & 71.01 & 74.57 & 78.53 & 0.0 & 1.7 & 1.1 & 99.3 & 65.7 & 0.1 \\
\hline 200 & 0.25 & 1 & 2.38 & 6.44 & 8.15 & 9.66 & 11.45 & 0.0 & 0.0 & 0.0 & 1.2 & 76.6 & 2.6 \\
\hline 200 & 0.25 & 2 & 9.15 & 15.40 & 17.63 & 19.90 & 22.38 & 0.0 & 0.0 & 0.0 & 18.2 & 8.0 & 0.4 \\
\hline 200 & 0.25 & 3 & 20.18 & 28.65 & 31.62 & 34.20 & 37.44 & 0.0 & 0.0 & 0.2 & 8.4 & 58.0 & 8.8 \\
\hline 200 & 0.25 & 4 & 35.09 & 45.46 & 48.85 & 52.08 & 56.03 & 0.0 & 0.0 & 4.6 & 72.8 & 0.8 & 3.7 \\
\hline 200 & 0.25 & 5 & 54.22 & 66.96 & 71.00 & 74.58 & 79.26 & 0.0 & 0.0 & 5.3 & 16.1 & 29.2 & 3.2 \\
\hline 200 & 0.5 & 1 & 2.29 & 6.35 & 7.93 & 9.40 & 11.23 & 0.0 & 0.0 & 0.0 & 0.2 & 1.4 & 0.0 \\
\hline 200 & 0.5 & 2 & 9.15 & 15.45 & 17.74 & 19.55 & 21.78 & 0.0 & 0.0 & 0.0 & 1.5 & 29.3 & 0.2 \\
\hline 200 & 0.5 & 3 & 20.13 & 28.51 & 31.11 & 33.39 & 36.59 & 0.0 & 0.0 & 0.0 & 17.4 & 70.2 & 62.6 \\
\hline 200 & 0.5 & 4 & 35.05 & 45.42 & 48.71 & 51.58 & 55.65 & 0.0 & 0.0 & 2.4 & 46.5 & 44.6 & 1.3 \\
\hline 200 & 0.5 & 5 & 54.07 & 66.74 & 70.73 & 74.41 & 79.18 & 0.0 & 0.0 & 0.2 & 47.3 & 29.3 & 37.5 \\
\hline 200 & 1 & 1 & 1.85 & 5.81 & 7.32 & 8.83 & 10.68 & 0.0 & 0.0 & 0.0 & 0.0 & 59.5 & 0.0 \\
\hline 200 & 1 & 2 & 8.89 & 15.06 & 17.25 & 19.36 & 21.90 & 0.0 & 0.0 & 0.0 & 0.0 & 4.0 & 45.5 \\
\hline 200 & 1 & 3 & 19.89 & 28.23 & 31.06 & 33.75 & 37.22 & 0.0 & 0.0 & 0.0 & 0.0 & 5.6 & 17.5 \\
\hline 200 & 1 & 4 & 34.80 & 45.44 & 48.90 & 52.18 & 55.93 & 0.0 & 0.0 & 0.0 & 0.2 & 80.3 & 91.5 \\
\hline 200 & 1 & 5 & 53.90 & 66.37 & 70.14 & 73.59 & 77.58 & 63.2 & 0.0 & 0.0 & 6.3 & 87.8 & 98.3 \\
\hline
\end{tabular}


Table 4: The table reports the Monte Carlo $j$ quantiles $x_{j}$ of the distribution of the LR statistics for model $\mathrm{M}_{\mu}^{*}$ under the null of $p-q$ common stochastic trends and in the presence of a local deterministic trend with parameter $\psi$. The table also reports the $p$ values of the Mann-Whitney test statistics for the test of identical distributions between the finite sample distribution of the LR statistic and its local-asymptotic approximation under the local alternative of near-stochastic trends parameterized by $\phi$. The number of Monte Carlo replications is 20,000 and the sample size $T$.

\begin{tabular}{|c|c|c|c|c|c|c|c|c|c|c|c|c|c|}
\hline \multirow[b]{2}{*}{$T$} & \multirow[b]{2}{*}{$\psi$} & \multirow[b]{2}{*}{$p-q$} & \multirow[b]{2}{*}{$x_{50}$} & \multirow[b]{2}{*}{$x_{90}$} & \multirow[b]{2}{*}{$x_{95}$} & \multirow[b]{2}{*}{$x_{97.5}$} & \multirow[b]{2}{*}{$x_{99}$} & \multicolumn{6}{|c|}{$p$-values of the Mann-Whitney test statistic in \% } \\
\hline & & & & & & & & $\phi=1$ & $\phi=.75$ & $\phi=.5$ & $\phi=.25$ & $\phi=0$ & $\phi=-0.25$ \\
\hline 50 & 0 & 1 & 5.30 & 9.52 & 11.00 & 12.46 & 14.07 & 0.0 & 0.0 & 0.0 & 0.0 & 0.0 & 0.0 \\
\hline 50 & 0 & 2 & 14.99 & 21.41 & 23.55 & 25.36 & 27.94 & 0.0 & 0.0 & 0.0 & 0.0 & 0.0 & 0.0 \\
\hline 50 & 0 & 3 & 28.61 & 37.28 & 40.00 & 42.57 & 45.54 & 0.0 & 0.0 & 0.0 & 0.0 & 0.0 & 0.0 \\
\hline 50 & 0 & 4 & 46.72 & 57.57 & 61.15 & 64.21 & 68.14 & 0.0 & 0.0 & 0.0 & 0.0 & 0.0 & 0.0 \\
\hline 50 & 0 & 5 & 69.20 & 83.14 & 87.31 & 91.87 & 97.18 & 41.3 & 0.0 & 0.7 & 0.0 & 0.0 & 0.9 \\
\hline 50 & 0.25 & 1 & 5.30 & 9.52 & 10.94 & 12.37 & 13.85 & 0.0 & 0.0 & 0.0 & 0.0 & 0.0 & 0.0 \\
\hline 50 & 0.25 & 2 & 14.99 & 21.35 & 23.52 & 25.34 & 27.65 & 0.0 & 0.0 & 0.0 & 0.0 & 0.0 & 0.0 \\
\hline 50 & 0.25 & 3 & 28.62 & 37.15 & 39.81 & 42.32 & 45.44 & 0.0 & 0.0 & 0.0 & 0.0 & 0.0 & 0.0 \\
\hline 50 & 0.25 & 4 & 46.54 & 57.77 & 61.25 & 64.55 & 68.06 & 0.0 & 0.0 & 0.0 & 0.0 & 0.0 & 0.0 \\
\hline 50 & 0.25 & 5 & 69.39 & 83.35 & 87.91 & 91.81 & 96.29 & 0.2 & 16.6 & 3.8 & 0.5 & 0.2 & 0.7 \\
\hline 50 & 0.5 & 1 & 5.30 & 9.43 & 10.85 & 12.23 & 14.10 & 0.0 & 0.0 & 0.0 & 0.0 & 0.0 & 0.0 \\
\hline 50 & 0.5 & 2 & 14.97 & 21.41 & 23.48 & 25.20 & 27.54 & 0.0 & 0.0 & 0.0 & 0.0 & 0.0 & 0.0 \\
\hline 50 & 0.5 & 3 & 28.71 & 37.29 & 40.00 & 42.85 & 45.67 & 0.0 & 0.0 & 0.0 & 0.0 & 0.0 & 0.0 \\
\hline 50 & 0.5 & 4 & 46.50 & 57.90 & 61.34 & 64.37 & 68.21 & 0.0 & 0.0 & 0.0 & 0.0 & 0.0 & 0.0 \\
\hline 50 & 0.5 & 5 & 69.47 & 83.26 & 87.47 & 91.61 & 96.42 & 0.0 & 0.7 & 8.0 & 0.0 & 0.0 & 0.1 \\
\hline 50 & 1 & 1 & 5.34 & 9.52 & 11.03 & 12.38 & 14.03 & 0.0 & 0.0 & 0.0 & 0.0 & 0.0 & 0.0 \\
\hline 50 & 1 & 2 & 14.98 & 21.38 & 23.45 & 25.43 & 27.85 & 0.0 & 0.0 & 0.0 & 0.0 & 0.0 & 0.0 \\
\hline 50 & 1 & 3 & 28.53 & 37.23 & 40.23 & 42.68 & 45.32 & 0.0 & 0.0 & 0.0 & 0.0 & 0.0 & 0.0 \\
\hline 50 & 1 & 4 & 46.67 & 57.95 & 61.63 & 65.07 & 68.83 & 0.0 & 0.0 & 0.0 & 0.0 & 0.0 & 0.0 \\
\hline 50 & 1 & 5 & 69.52 & 83.25 & 87.91 & 91.73 & 95.43 & 0.0 & 0.0 & 0.2 & 2.3 & 1.0 & 0.0 \\
\hline 100 & 0 & 1 & 5.50 & 10.14 & 11.76 & 13.44 & 15.40 & 16.1 & 0.6 & 11.3 & 1.6 & 0.7 & 0.0 \\
\hline 100 & 0 & 2 & 15.40 & 22.41 & 24.77 & 27.22 & 29.72 & 0.3 & 0.0 & 0.0 & 0.0 & 0.0 & 0.0 \\
\hline 100 & 0 & 3 & 29.40 & 38.52 & 41.63 & 44.19 & 47.22 & 0.0 & 0.0 & 0.0 & 0.0 & 0.0 & 0.0 \\
\hline 100 & 0 & 4 & 47.35 & 58.81 & 62.27 & 65.76 & 69.68 & 0.1 & 0.0 & 0.2 & 0.0 & 0.0 & 0.0 \\
\hline 100 & 0 & 5 & 69.80 & 83.76 & 87.94 & 92.31 & 97.19 & 77.3 & 19.1 & 20.0 & 0.3 & 6.9 & 9.2 \\
\hline 100 & 0.25 & 1 & 5.49 & 10.08 & 11.73 & 13.24 & 15.31 & 0.5 & 0.1 & 4.7 & 0.1 & 1.0 & 0.1 \\
\hline 100 & 0.25 & 2 & 15.49 & 22.37 & 24.87 & 27.04 & 29.62 & 0.0 & 0.0 & 0.1 & 0.0 & 0.0 & 0.5 \\
\hline 100 & 0.25 & 3 & 29.36 & 38.63 & 41.45 & 44.26 & 47.97 & 0.0 & 0.0 & 0.0 & 0.0 & 0.0 & 0.0 \\
\hline 100 & 0.25 & 4 & 47.44 & 58.78 & 62.21 & 65.43 & 69.75 & 0.0 & 0.0 & 0.0 & 0.0 & 0.0 & 0.1 \\
\hline 100 & 0.25 & 5 & 69.72 & 83.10 & 87.91 & 91.47 & 96.01 & 8.3 & 14.4 & 6.8 & 0.0 & 0.8 & 36.5 \\
\hline 100 & 0.5 & 1 & 5.49 & 10.14 & 11.82 & 13.40 & 15.33 & 19.1 & 0.3 & 2.6 & 0.5 & 5.8 & 1.0 \\
\hline 100 & 0.5 & 2 & 15.57 & 22.47 & 24.94 & 26.92 & 29.80 & 0.0 & 0.0 & 0.0 & 0.0 & 0.0 & 0.1 \\
\hline 100 & 0.5 & 3 & 29.28 & 38.54 & 41.58 & 44.09 & 47.09 & 0.0 & 0.0 & 0.0 & 0.0 & 0.0 & 0.5 \\
\hline 100 & 0.5 & 4 & 47.31 & 58.88 & 62.24 & 65.37 & 69.64 & 0.0 & 0.6 & 0.0 & 0.0 & 0.0 & 0.0 \\
\hline 100 & 0.5 & 5 & 69.62 & 83.48 & 87.84 & 91.18 & 95.73 & 15.0 & 10.2 & 0.6 & 0.1 & 4.0 & 20.2 \\
\hline 100 & 1 & 1 & 5.56 & 10.09 & 11.74 & 13.31 & 15.40 & 7.4 & 1.3 & 0.6 & 0.0 & 0.0 & 0.0 \\
\hline 100 & 1 & 2 & 15.60 & 22.44 & 24.70 & 26.80 & 29.41 & 0.0 & 0.0 & 0.0 & 0.1 & 0.0 & 0.0 \\
\hline 100 & 1 & 3 & 29.36 & 38.73 & 41.65 & 44.23 & 47.61 & 0.0 & 0.0 & 0.0 & 0.1 & 0.0 & 0.0 \\
\hline 100 & 1 & 4 & 47.36 & 58.45 & 61.98 & 65.21 & 69.76 & 0.0 & 0.0 & 0.0 & 0.1 & 0.0 & 0.6 \\
\hline 100 & 1 & 5 & 69.61 & 83.18 & 87.24 & 90.88 & 95.18 & 0.0 & 18.7 & 4.8 & 0.9 & 0.1 & 5.6 \\
\hline 200 & 0 & 1 & 5.58 & 10.41 & 12.21 & 14.07 & 16.07 & 0.1 & 46.9 & 5.2 & 43.5 & 68.9 & 11.2 \\
\hline 200 & 0 & 2 & 15.67 & 22.87 & 25.31 & 27.39 & 30.44 & 0.9 & 12.1 & 13.6 & 25.0 & 4.6 & 0.0 \\
\hline 200 & 0 & 3 & 29.69 & 39.19 & 42.26 & 45.19 & 48.77 & 0.0 & 12.9 & 0.2 & 81.8 & 0.7 & 1.3 \\
\hline 200 & 0 & 4 & 47.76 & 59.54 & 62.98 & 66.23 & 70.21 & 9.5 & 76.7 & 40.3 & 1.0 & 30.7 & 0.7 \\
\hline 200 & 0 & 5 & 70.04 & 83.91 & 88.36 & 91.87 & 96.16 & 54.8 & 14.0 & 21.8 & 0.7 & 58.0 & 1.2 \\
\hline 200 & 0.25 & 1 & 5.63 & 10.47 & 12.20 & 13.84 & 15.78 & 0.0 & 30.4 & 0.9 & 56.6 & 24.9 & 77.5 \\
\hline 200 & 0.25 & 2 & 15.68 & 22.78 & 25.28 & 27.57 & 30.19 & 0.7 & 30.2 & 1.3 & 5.7 & 15.8 & 5.0 \\
\hline 200 & 0.25 & 3 & 29.77 & 39.54 & 42.51 & 45.57 & 49.50 & 65.9 & 20.9 & 15.2 & 41.6 & 11.8 & 0.0 \\
\hline 200 & 0.25 & 4 & 47.71 & 59.31 & 62.80 & 65.92 & 70.46 & 0.9 & 5.0 & 0.5 & 4.8 & 0.3 & 22.8 \\
\hline 200 & 0.25 & 5 & 70.02 & 83.74 & 88.19 & 92.17 & 97.02 & 25.2 & 93.1 & 37.8 & 91.8 & 10.7 & 77.6 \\
\hline 200 & 0.5 & 1 & 5.63 & 10.36 & 12.09 & 13.52 & 15.41 & 14.1 & 39.3 & 55.2 & 7.9 & 96.2 & 14.4 \\
\hline 200 & 0.5 & 2 & 15.77 & 22.74 & 25.14 & 27.54 & 30.26 & 1.3 & 22.8 & 4.8 & 3.2 & 22.1 & 1.2 \\
\hline 200 & 0.5 & 3 & 29.70 & 39.04 & 42.26 & 45.31 & 48.18 & 15.5 & 1.7 & 0.1 & 8.6 & 14.6 & 0.1 \\
\hline 200 & 0.5 & 4 & 47.73 & 59.28 & 62.90 & 66.19 & 70.19 & 54.0 & 43.5 & 17.0 & 11.3 & 0.5 & 0.2 \\
\hline 200 & 0.5 & 5 & 69.85 & 83.93 & 88.59 & 92.61 & 97.90 & 22.2 & 9.8 & 27.8 & 49.3 & 51.6 & 30.5 \\
\hline 200 & 1 & 1 & 5.59 & 10.39 & 12.14 & 14.06 & 16.26 & 12.2 & 69.7 & 12.3 & 84.1 & 20.8 & 0.1 \\
\hline 200 & 1 & 2 & 15.66 & 22.67 & 25.08 & 27.38 & 30.46 & 0.0 & 0.0 & 2.1 & 0.6 & 19.0 & 6.3 \\
\hline 200 & 1 & 3 & 29.73 & 39.06 & 42.54 & 45.42 & 48.63 & 3.2 & 0.0 & 46.7 & 2.4 & 0.4 & 7.6 \\
\hline 200 & 1 & 4 & 47.64 & 59.34 & 63.09 & 66.37 & 70.49 & 0.0 & 0.1 & 0.4 & 5.3 & 6.1 & 11.9 \\
\hline 200 & 1 & 5 & 69.96 & 83.38 & 87.84 & 91.53 & 95.66 & 0.1 & 15.7 & 35.0 & 3.5 & 0.1 & 2.9 \\
\hline
\end{tabular}


Table 5: The table reports the Monte Carlo $j$ quantiles $x_{j}$ of the distribution of the LR statistics for model $\mathrm{M}_{\tau}$ under the null of $p-q$ common stochastic trends and in the presence of a local deterministic trend with parameter $\psi$. The table also reports the $p$ values of the Mann-Whitney test statistics for the test of identical distributions between the finite sample distribution of the LR statistic and its local-asymptotic approximation under the local alternative of near-stochastic trends parameterized by $\phi$. The number of Monte Carlo replications is 20,000 and the sample size $T$.

\begin{tabular}{|c|c|c|c|c|c|c|c|c|c|c|c|c|c|}
\hline \multirow[b]{2}{*}{$T$} & \multirow[b]{2}{*}{$\psi$} & \multirow[b]{2}{*}{$p-q$} & \multirow[b]{2}{*}{$x_{50}$} & \multirow[b]{2}{*}{$x_{90}$} & \multirow[b]{2}{*}{$x_{95}$} & \multirow[b]{2}{*}{$x_{97.5}$} & \multirow[b]{2}{*}{$x_{99}$} & \multicolumn{6}{|c|}{$p$-values of the Mann-Whitney test statistic in \% } \\
\hline & & & & & & & & $\phi=1$ & $\phi=.75$ & $\phi=.5$ & $\phi=.25$ & $\phi=0$ & $\phi=-0.25$ \\
\hline 50 & 0 & 1 & 4.37 & 8.66 & 10.35 & 11.89 & 13.72 & 0.0 & 0.0 & 0.0 & 0.0 & 0.0 & 0.0 \\
\hline 50 & 0 & 2 & 13.00 & 19.67 & 21.78 & 23.83 & 26.35 & 0.0 & 0.0 & 0.0 & 0.0 & 0.0 & 0.0 \\
\hline 50 & 0 & 3 & 25.68 & 34.39 & 37.02 & 39.59 & 42.58 & 0.0 & 0.0 & 0.0 & 0.0 & 0.0 & 0.0 \\
\hline 50 & 0 & 4 & 42.71 & 53.78 & 57.09 & 60.36 & 64.39 & 0.0 & 0.0 & 0.0 & 0.0 & 0.0 & 0.0 \\
\hline 50 & 0 & 5 & 64.11 & 77.75 & 82.35 & 86.78 & 91.62 & 1.4 & 0.0 & 0.0 & 0.0 & 0.0 & 0.0 \\
\hline 50 & 0.25 & 1 & 4.38 & 8.77 & 10.34 & 11.67 & 13.46 & 0.0 & 0.0 & 0.0 & 0.0 & 0.0 & 0.0 \\
\hline 50 & 0.25 & 2 & 12.96 & 19.54 & 21.66 & 23.73 & 26.00 & 0.0 & 0.0 & 0.0 & 0.0 & 0.0 & 0.0 \\
\hline 50 & 0.25 & 3 & 25.59 & 34.49 & 37.19 & 39.58 & 42.41 & 0.0 & 0.0 & 0.0 & 0.0 & 0.0 & 0.0 \\
\hline 50 & 0.25 & 4 & 42.45 & 53.90 & 57.33 & 60.37 & 64.07 & 0.0 & 0.0 & 0.0 & 0.0 & 0.0 & 0.0 \\
\hline 50 & 0.25 & 5 & 64.33 & 78.20 & 82.59 & 86.69 & 91.24 & 0.0 & 0.0 & 0.0 & 0.0 & 0.0 & 0.0 \\
\hline 50 & 0.5 & 1 & 4.38 & 8.63 & 10.14 & 11.64 & 13.63 & 0.0 & 0.0 & 0.0 & 0.0 & 0.0 & 0.0 \\
\hline 50 & 0.5 & 2 & 13.04 & 19.73 & 21.73 & 23.73 & 25.89 & 0.0 & 0.0 & 0.0 & 0.0 & 0.0 & 0.0 \\
\hline 50 & 0.5 & 3 & 25.67 & 34.48 & 37.38 & 39.89 & 43.27 & 0.0 & 0.0 & 0.0 & 0.0 & 0.0 & 0.0 \\
\hline 50 & 0.5 & 4 & 42.46 & 53.89 & 57.26 & 60.31 & 64.07 & 0.0 & 0.0 & 0.0 & 0.0 & 0.0 & 0.0 \\
\hline 50 & 0.5 & 5 & 64.30 & 78.22 & 82.59 & 86.29 & 90.66 & 0.0 & 0.0 & 0.0 & 0.0 & 0.0 & 0.0 \\
\hline 50 & 1 & 1 & 4.40 & 8.72 & 10.37 & 11.83 & 13.61 & 3.0 & 0.0 & 0.0 & 0.0 & 0.0 & 0.0 \\
\hline 50 & 1 & 2 & 12.98 & 19.64 & 21.84 & 23.96 & 26.39 & 0.0 & 0.0 & 0.0 & 0.0 & 0.0 & 0.0 \\
\hline 50 & 1 & 3 & 25.56 & 34.28 & 37.14 & 39.95 & 43.03 & 0.0 & 0.0 & 0.0 & 0.0 & 0.0 & 0.0 \\
\hline 50 & 1 & 4 & 42.62 & 54.07 & 57.61 & 60.88 & 64.70 & 0.0 & 0.0 & 0.0 & 0.0 & 0.0 & 0.0 \\
\hline 50 & 1 & 5 & 64.20 & 78.26 & 82.56 & 86.56 & 91.09 & 17.7 & 0.0 & 0.0 & 6.5 & 0.0 & 0.0 \\
\hline 100 & 0 & 1 & 4.56 & 9.25 & 11.10 & 12.74 & 14.73 & 12.7 & 0.1 & 0.0 & 13.3 & 3.1 & 0.0 \\
\hline 100 & 0 & 2 & 13.45 & 20.53 & 22.84 & 25.09 & 27.92 & 0.0 & 0.0 & 0.0 & 0.0 & 0.0 & 0.0 \\
\hline 100 & 0 & 3 & 26.48 & 35.67 & 38.50 & 41.32 & 44.29 & 0.0 & 0.0 & 0.0 & 0.0 & 0.0 & 0.0 \\
\hline 100 & 0 & 4 & 43.32 & 54.93 & 58.45 & 61.78 & 65.74 & 0.0 & 0.0 & 0.0 & 0.0 & 0.0 & 0.0 \\
\hline 100 & 0 & 5 & 64.73 & 78.65 & 82.82 & 87.09 & 92.41 & 14.8 & 0.1 & 1.4 & 0.0 & 7.2 & 1.1 \\
\hline 100 & 0.25 & 1 & 4.54 & 9.22 & 11.01 & 12.64 & 14.28 & 0.0 & 0.3 & 4.2 & 0.0 & 0.4 & 0.0 \\
\hline 100 & 0.25 & 2 & 13.53 & 20.60 & 23.22 & 25.37 & 28.10 & 0.0 & 0.0 & 0.0 & 0.0 & 0.0 & 0.4 \\
\hline 100 & 0.25 & 3 & 26.36 & 35.66 & 38.64 & 41.29 & 45.15 & 0.0 & 0.0 & 0.0 & 0.0 & 0.0 & 0.0 \\
\hline 100 & 0.25 & 4 & 43.40 & 54.86 & 58.41 & 61.58 & 65.58 & 0.0 & 0.0 & 0.0 & 0.0 & 0.0 & 0.0 \\
\hline 100 & 0.25 & 5 & 64.69 & 78.07 & 82.78 & 86.45 & 91.75 & 1.4 & 4.3 & 0.7 & 0.0 & 0.1 & 15.9 \\
\hline 100 & 0.5 & 1 & 4.59 & 9.24 & 11.01 & 12.73 & 14.56 & 38.2 & 0.0 & 0.9 & 0.0 & 0.3 & 0.1 \\
\hline 100 & 0.5 & 2 & 13.65 & 20.71 & 23.17 & 25.30 & 28.00 & 0.0 & 0.0 & 0.0 & 0.0 & 0.0 & 0.0 \\
\hline 100 & 0.5 & 3 & 26.32 & 35.51 & 38.45 & 41.20 & 44.67 & 0.0 & 0.0 & 0.0 & 0.0 & 0.0 & 0.0 \\
\hline 100 & 0.5 & 4 & 43.23 & 54.68 & 58.31 & 61.52 & 65.04 & 0.0 & 0.0 & 0.0 & 0.0 & 0.0 & 0.0 \\
\hline 100 & 0.5 & 5 & 64.53 & 78.39 & 82.89 & 86.12 & 91.06 & 15.6 & 0.9 & 0.6 & 0.0 & 3.1 & 0.4 \\
\hline 100 & 1 & 1 & 4.57 & 9.26 & 11.00 & 12.57 & 14.73 & 99.8 & 88.3 & 0.5 & 0.2 & 0.0 & 0.0 \\
\hline 100 & 1 & 2 & 13.60 & 20.64 & 23.00 & 25.09 & 27.76 & 17.7 & 0.5 & 0.1 & 0.1 & 0.0 & 0.0 \\
\hline 100 & 1 & 3 & 26.36 & 35.61 & 38.76 & 41.50 & 44.79 & 32.1 & 0.0 & 0.2 & 0.1 & 0.0 & 0.0 \\
\hline 100 & 1 & 4 & 43.27 & 54.70 & 58.18 & 61.46 & 65.63 & 41.9 & 0.0 & 0.0 & 0.0 & 0.0 & 0.7 \\
\hline 100 & 1 & 5 & 64.57 & 78.17 & 82.26 & 86.10 & 91.02 & 75.5 & 58.4 & 2.5 & 1.5 & 0.3 & 0.0 \\
\hline 200 & 0 & 1 & 4.65 & 9.55 & 11.32 & 13.31 & 15.39 & 1.0 & 14.7 & 3.1 & 22.5 & 55.2 & 9.4 \\
\hline 200 & 0 & 2 & 13.67 & 21.00 & 23.48 & 25.62 & 28.59 & 0.0 & 2.0 & 3.8 & 10.7 & 10.0 & 0.0 \\
\hline 200 & 0 & 3 & 26.76 & 36.17 & 39.45 & 42.25 & 45.99 & 0.0 & 5.3 & 0.0 & 22.0 & 0.1 & 1.0 \\
\hline 200 & 0 & 4 & 43.66 & 55.47 & 59.41 & 62.48 & 66.62 & 0.1 & 40.8 & 1.3 & 1.3 & 98.7 & 0.4 \\
\hline 200 & 0 & 5 & 65.11 & 78.73 & 83.12 & 87.10 & 91.46 & 5.3 & 0.2 & 7.1 & 0.2 & 33.0 & 0.0 \\
\hline 200 & 0.25 & 1 & 4.73 & 9.59 & 11.37 & 13.06 & 15.13 & 0.0 & 15.0 & 0.0 & 14.7 & 10.9 & 23.3 \\
\hline 200 & 0.25 & 2 & 13.71 & 20.93 & 23.32 & 25.77 & 28.22 & 5.3 & 98.6 & 3.5 & 7.3 & 8.3 & 11.8 \\
\hline 200 & 0.25 & 3 & 26.75 & 36.34 & 39.47 & 42.09 & 46.38 & 1.9 & 1.2 & 5.5 & 43.3 & 57.7 & 0.3 \\
\hline 200 & 0.25 & 4 & 43.78 & 55.36 & 58.81 & 61.86 & 65.90 & 0.0 & 1.4 & 0.0 & 36.6 & 0.8 & 2.7 \\
\hline 200 & 0.25 & 5 & 64.91 & 78.66 & 83.11 & 87.01 & 91.30 & 0.3 & 16.3 & 6.0 & 76.7 & 22.0 & 42.5 \\
\hline 200 & 0.5 & 1 & 4.70 & 9.55 & 11.16 & 12.77 & 14.72 & 37.7 & 11.9 & 28.2 & 2.6 & 54.3 & 24.9 \\
\hline 200 & 0.5 & 2 & 13.75 & 20.88 & 23.26 & 25.49 & 28.42 & 7.8 & 0.3 & 14.5 & 0.5 & 14.0 & 11.5 \\
\hline 200 & 0.5 & 3 & 26.74 & 36.25 & 39.48 & 42.19 & 45.64 & 47.3 & 0.0 & 1.5 & 3.0 & 2.8 & 0.8 \\
\hline 200 & 0.5 & 4 & 43.65 & 55.33 & 58.83 & 61.94 & 65.91 & 16.6 & 25.7 & 35.8 & 5.1 & 0.0 & 0.3 \\
\hline 200 & 0.5 & 5 & 64.76 & 78.97 & 83.05 & 87.72 & 92.39 & 42.8 & 2.5 & 0.7 & 7.3 & 38.4 & 37.3 \\
\hline 200 & 1 & 1 & 4.64 & 9.54 & 11.35 & 13.17 & 15.34 & 4.6 & 82.0 & 45.0 & 71.2 & 20.2 & 0.2 \\
\hline 200 & 1 & 2 & 13.69 & 20.84 & 23.31 & 25.40 & 28.33 & 38.4 & 0.4 & 4.4 & 1.3 & 25.8 & 0.1 \\
\hline 200 & 1 & 3 & 26.67 & 36.23 & 39.47 & 42.48 & 45.74 & 1.7 & 0.9 & 29.9 & 8.5 & 1.2 & 1.5 \\
\hline 200 & 1 & 4 & 43.71 & 55.32 & 58.96 & 62.53 & 66.44 & 74.4 & 18.9 & 1.5 & 14.5 & 0.3 & 2.5 \\
\hline 200 & 1 & 5 & 64.94 & 78.21 & 82.41 & 86.37 & 90.44 & 12.3 & 58.2 & 29.0 & 1.5 & 0.0 & 2.2 \\
\hline
\end{tabular}



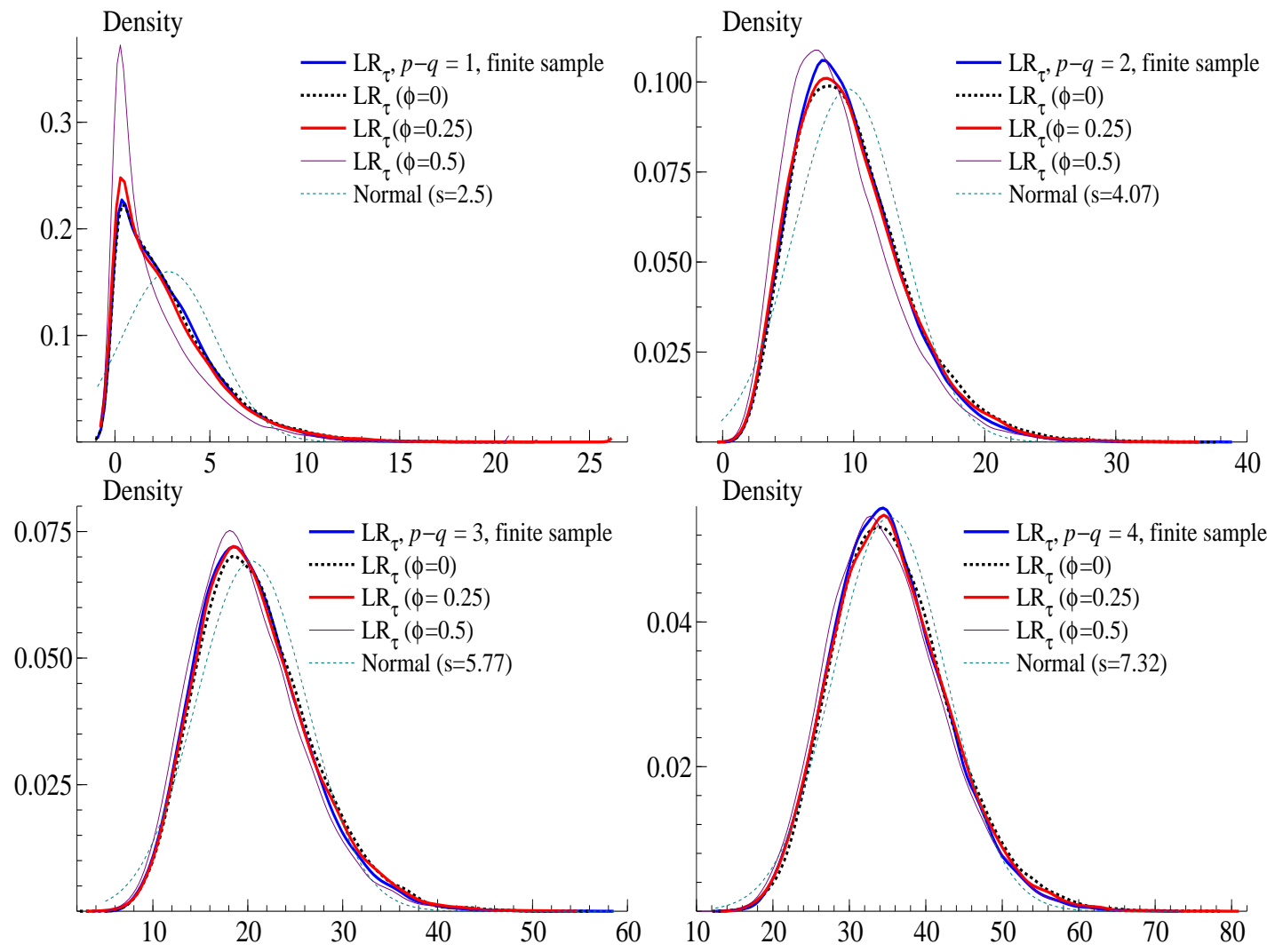

Figure 1: Smooth kernel estimation of the densities of the finite sample distribution of the $\mathrm{LR}_{\tau}$ statistic and corresponding local asymptotic approximations. The sample size is $T=100$, the deterministic component is parameterized as $T^{-1 / 2}(\psi, 0, \ldots)^{\prime}$ where $\psi=0.5$. $(p-q)$ denotes the number of common stochastic trends. Local asymptotic approximations are denoted according to the parameter of the nearstochastic trends $\phi$. A normal approximation using the same mean and variance as the finite sample distribution of $\mathrm{LR}_{\tau}$ is also drawn. The number of observations used in the local approximation and the number of Monte Carlo replications are both 20,000 . 

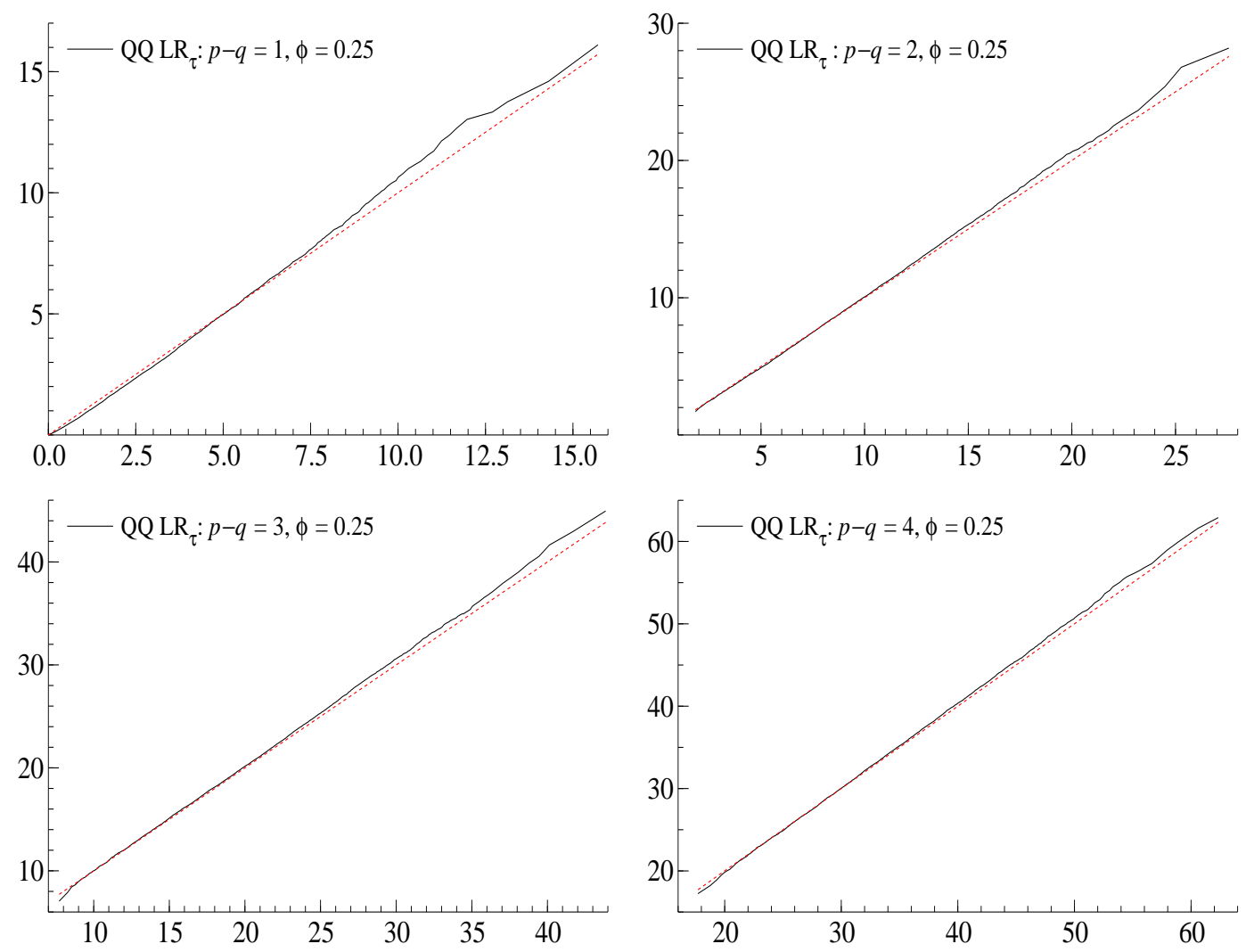

Figure 2: QQ plot of the densities of the finite sample $(T=100, \psi=.5)$ distributions of $\mathrm{LR}_{\tau}$ and corresponding local asymptotic approximations $(\phi=.25) \cdot p-q$ denotes the number of common stochastic trends. The number of observations used in the local approximation and the number of Monte Carlo replications are both 20,000. 


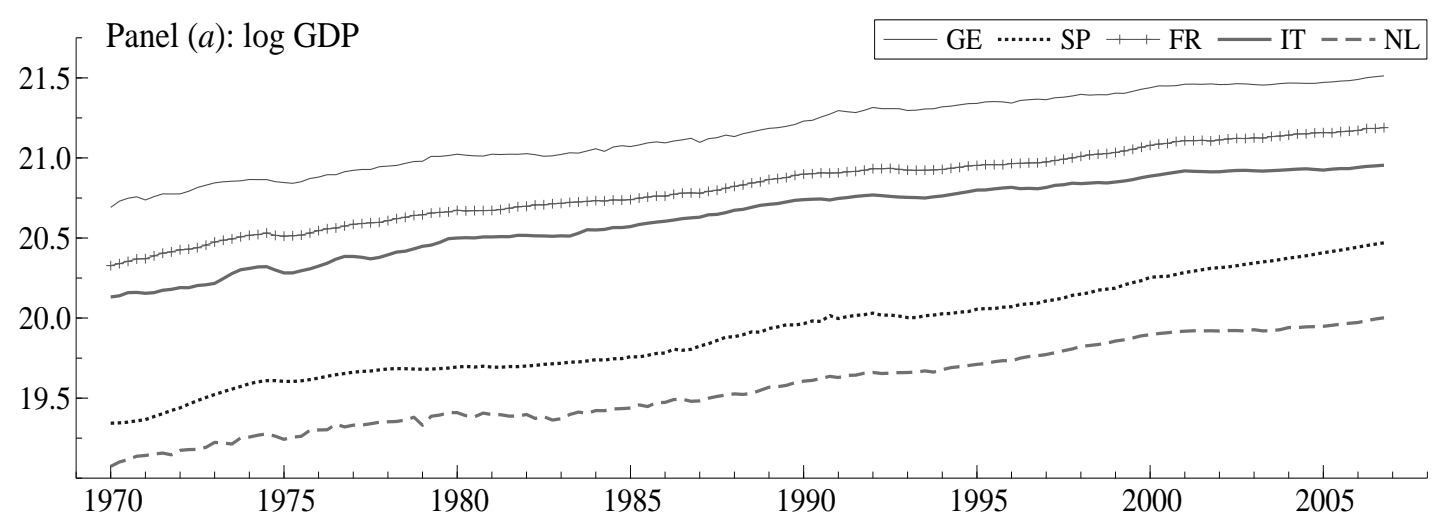

Panel $(b): \log$ GDP in deviation from trend

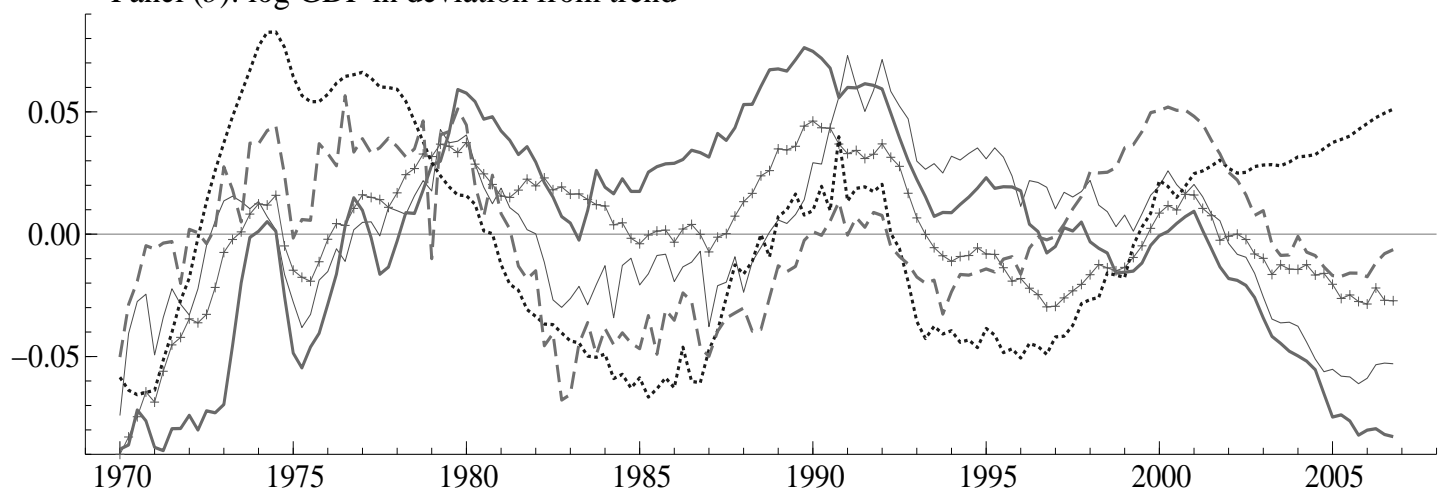

Figure 3: Panel (a): Natural logarithm of the quarterly Gross Domestic Product (in constant prices) of France (FR), Germany (GE), Italy (IT), the Netherlands (NL) and Spain (SP). Panel (b): the same variables in deviation from an estimated linear trend. 


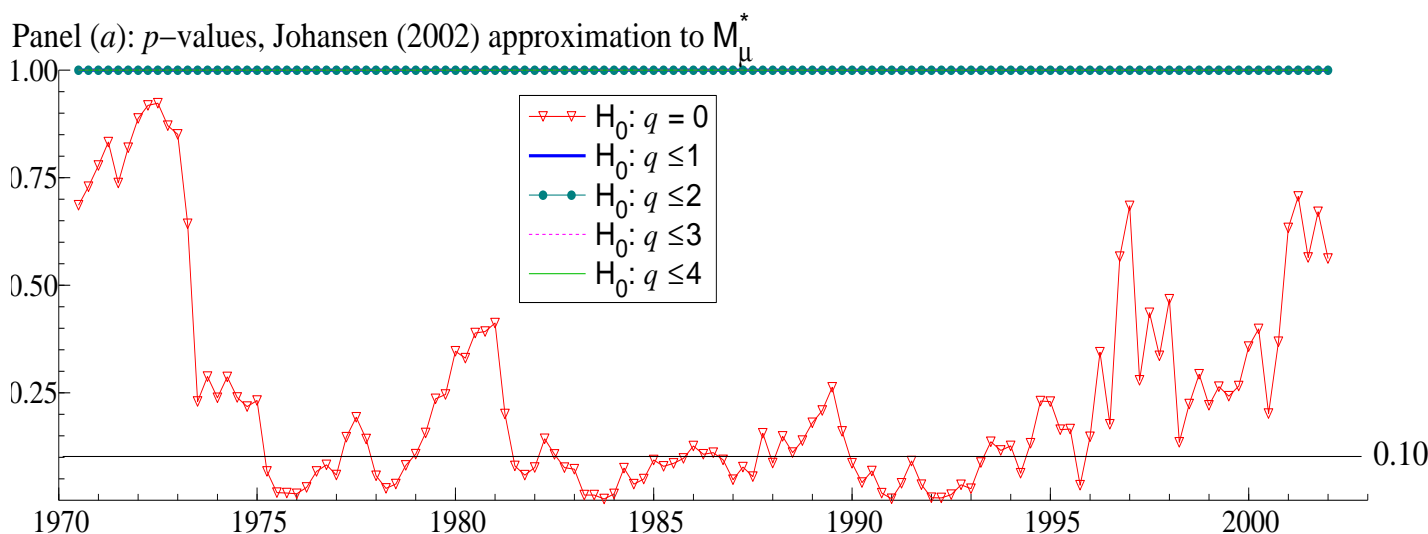

Panel (b): p-values, Johansen (2002) approximation to $\mathrm{M}_{\tau}$

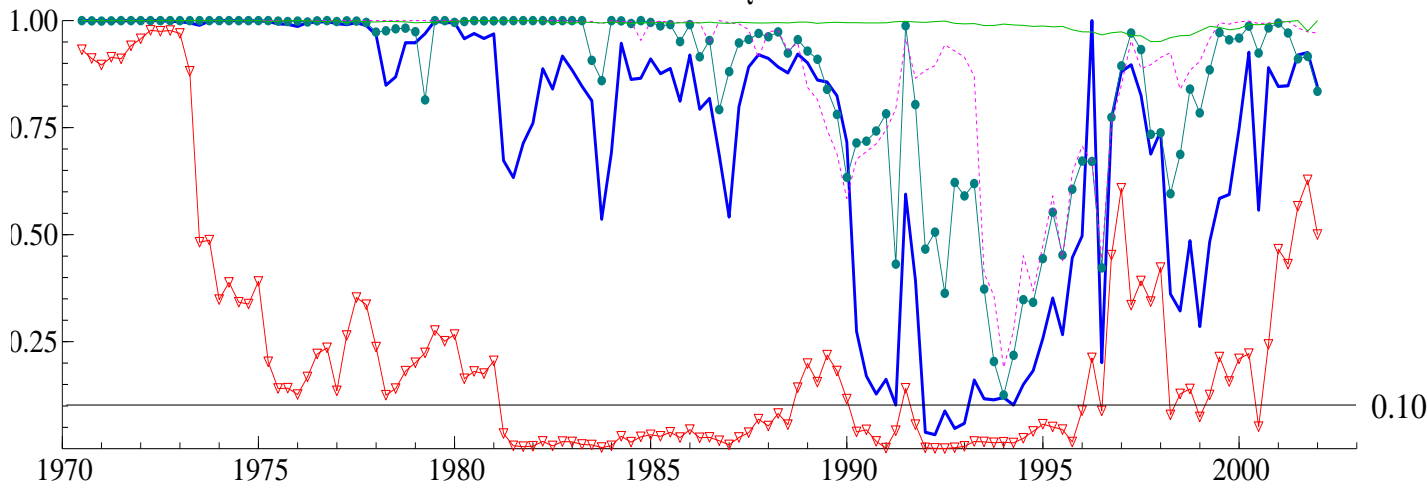

Figure 4: $p$-values of the Likelihood Ratio test of at most $q$ cointegrating relations for models $\mathbf{M}_{\mu}^{*}$ (Panel $(a)$ ) and $\mathbf{M}_{\tau}($ Panel $(b)$ ), respectively with a deterministic trend restricted to lie within the cointegrating space and entering unrestrictedly. The distributions under the null are computed using the finite sample correction in Johansen $(2002 \mathrm{a}, \mathrm{b})$. The dates on the horizontal axis report the starting observation of the sample used in the test, the end-point is held constant at 2006(4). The solid horizontal line represents the $10 \%$ level. 


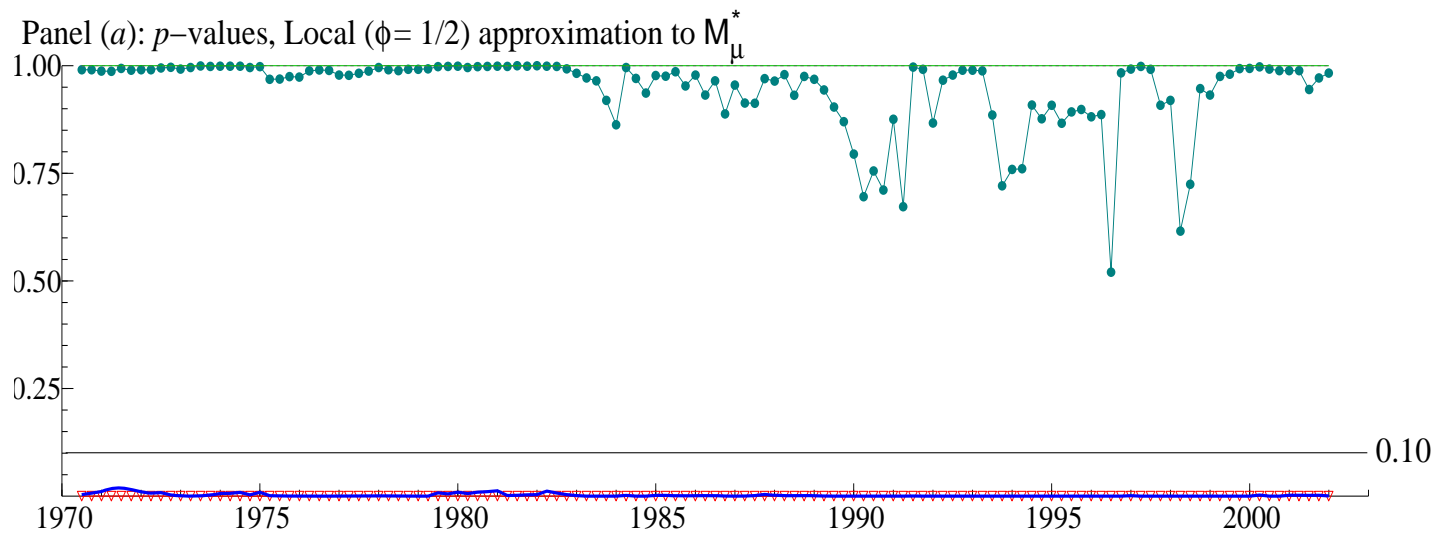

Panel $(b)$ : $p$-values, Local $(\phi=1 / 2)$ approximation to $M_{\tau}$

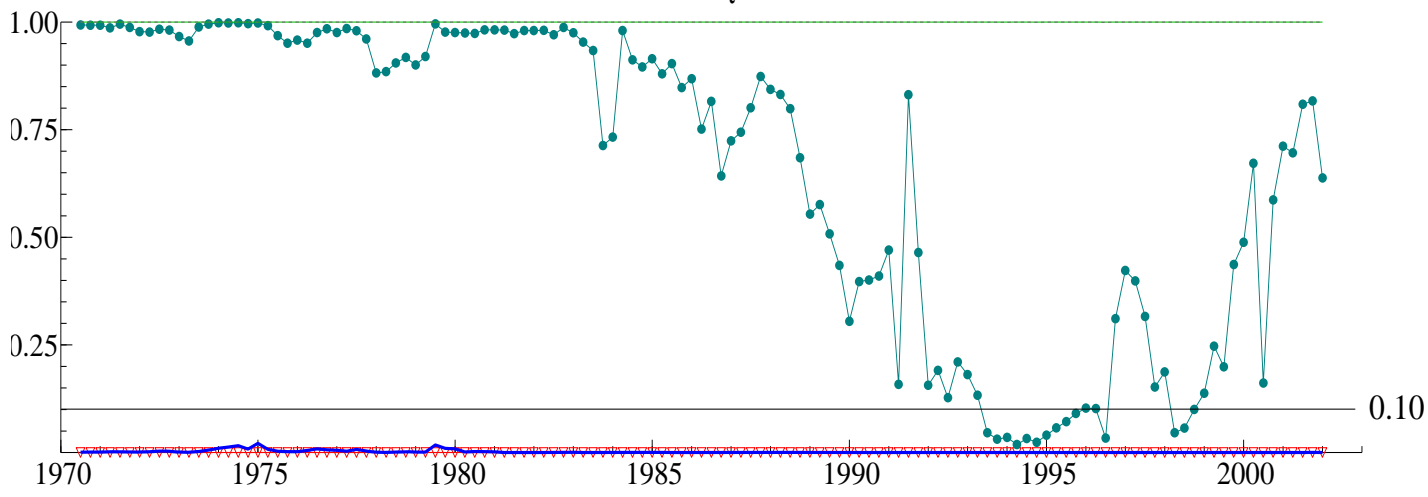

Figure 5: $p$-values of the Likelihood Ratio test of at most $q$ cointegrating relations for models $\mathbf{M}_{\mu}^{*}$ (Panel $(a)$ ) and $\mathbf{M}_{\tau}$ (Panel $(b)$ ) respectively with a deterministic trend restricted to lie within the cointegrating space and entering unrestrictedly. The distributions are the local asymptotic simulated distribution using $\phi=1 / 2$ where we impose that $\psi=0$. The dates on the horizontal axis report the starting observation of the sample used in the test, the end-point is held constant at 2006(4). The solid horizontal line represents the $10 \%$ level. 
Panel $(a)$ : $p$-values, Local $(\phi=0)$ approximation to $\mathrm{M}_{\mu}^{*}$, with estimated $\psi$.
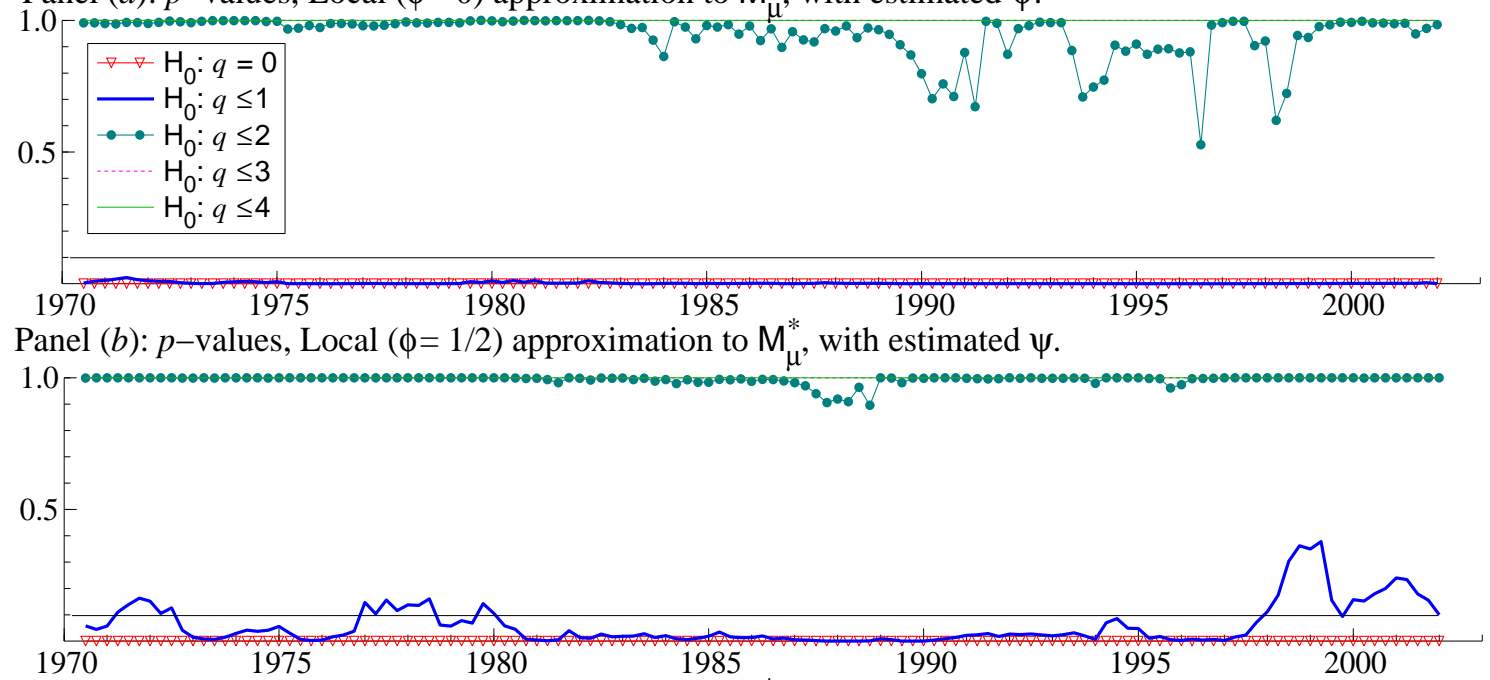

Panel $(c)$ : $p$-values, Local $(\phi=1)$ approximation to $\mathrm{M}_{\mu}^{*}$, with estimated $\psi$.

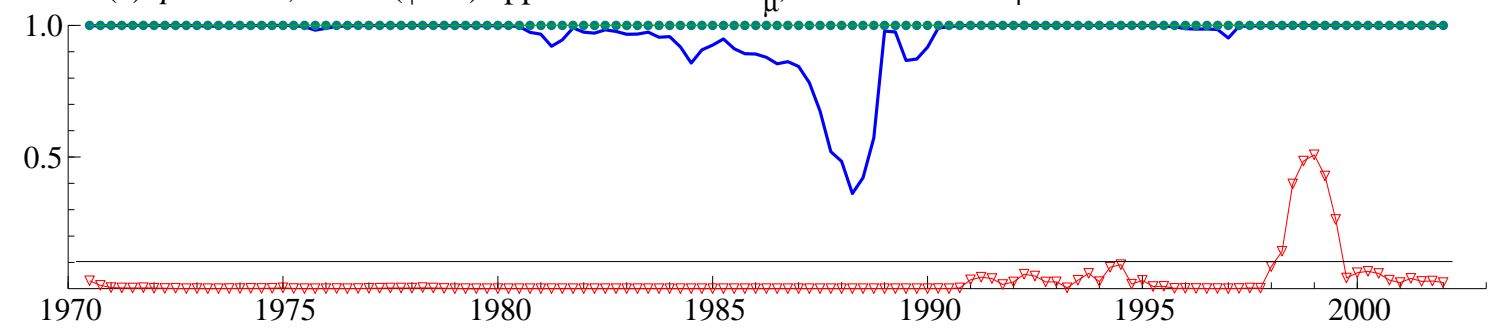

Figure 6: $p$-values of the Likelihood Ratio test of at most $q$ cointegrating relations for model $\mathrm{M}_{\mu}^{*}$ with a deterministic trend restricted to lie within the cointegrating space. The distributions under the null are the local asymptotic simulated distribution where the linear trend coefficient is estimated via OLS. Panels $(a)$ to $(c)$ use values of $\phi=0,1 / 2$ and 1 . The dates on the horizontal axis report the starting observation of the sample used in the test, the end-point is held constant at 2006(4). The solid horizontal line represents the $10 \%$ level. 
Panel $(a)$ : $p$-values, Local $(\phi=0)$ approximation to $\mathrm{M}_{\tau}$, with estimated $\psi$.

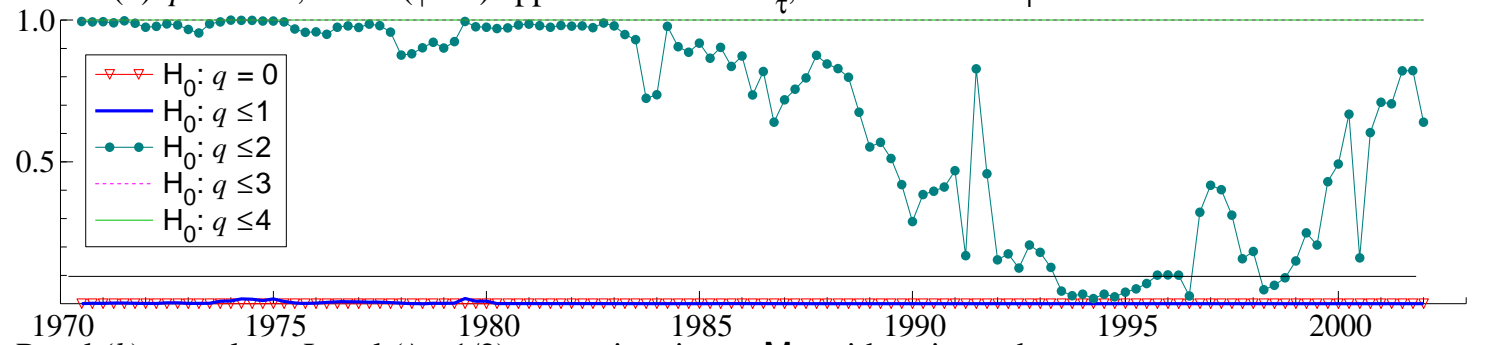

Panel $(b)$ : $p$-values, Local $(\phi=1 / 2)$ approximation to $\mathrm{M}_{\tau}$, with estimated $\psi$.

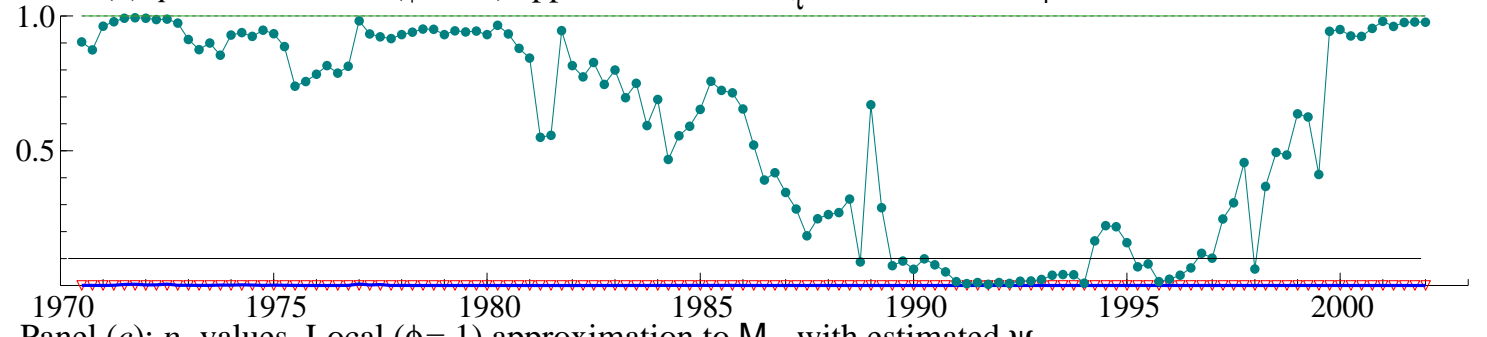

Panel $(c)$ : $p$-values, Local $(\phi=1)$ approximation to $\mathrm{M}_{\tau}$, with estimated $\psi$.

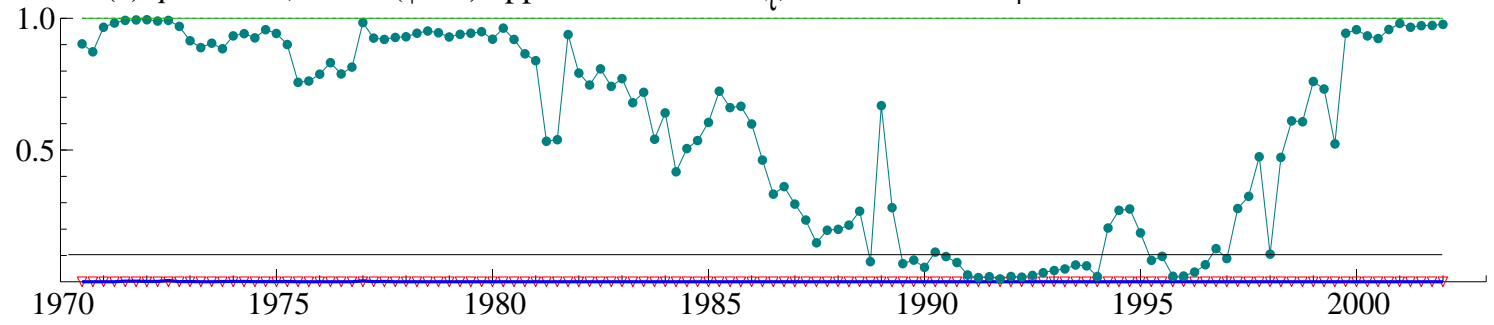

Figure 7: $p$-values of the Likelihood Ratio test of at most $q$ cointegrating relations for model $\mathrm{M}_{\tau}$ with a deterministic trend entering the cointegrating space unrestrictedly. The distributions under the null are the local asymptotic simulated distribution where the linear trend coefficient is estimated via OLS. Panels $(a)$ to $(c)$ use values of $\phi=0,1 / 2$ and 1 . The dates on the horizontal axis report the starting observation of the sample used in the test, the end-point is held constant at 2006(4). The solid horizontal line represents the $10 \%$ level. 

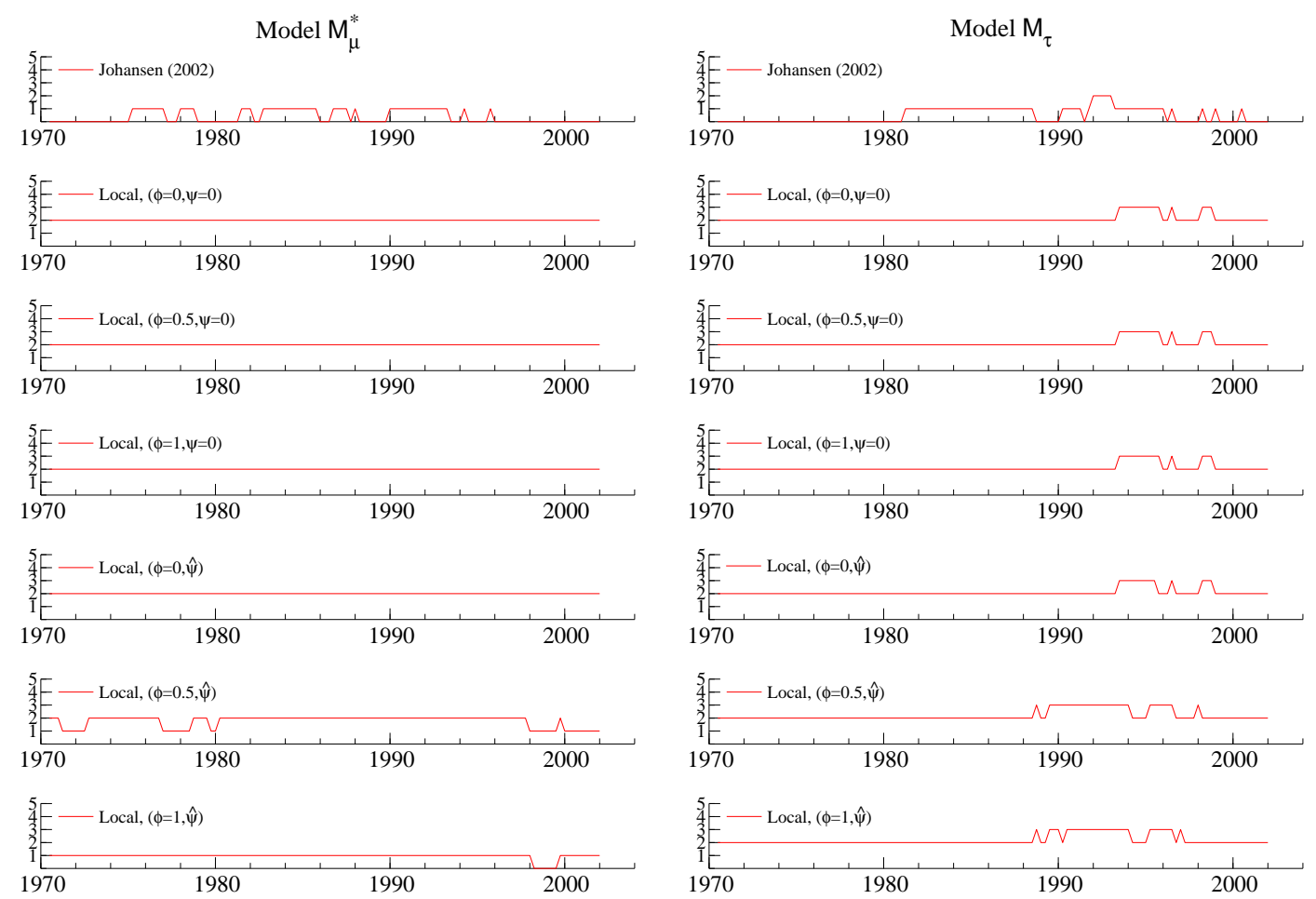

Figure 8: The figure reports the selected rank of cointegration under models $\mathrm{M}_{\mu}^{*}$ (left column) and $\mathrm{M}_{\tau}$ (right column) according to the LR tests at a 0.10 significance level. The distributions considered are the Johansen (2002) finite sample approximation (first row) and the local approximations suggested in the paper for various values of the parameters $\phi$ and $\psi$. Rows corresponding to $\widehat{\psi}$ use the parametric bootstrap technique described in section 5 . 
ESSEC Business School Avenue Bernard Hirsch BP 50105

95021 Cergy-Pontoise Cedex France

Tél. +33(0)134433000

$\mathrm{Fax}+33(0) 134433001$

www.essec.fr

\section{ESSEC Executive Education}

CNIT BP 230

92053 Paris-La Défense

France

Tél. +33(0)146924900

Fax +33(0)1 46924990

http:l/formation.essec.fr

ESSEC Business School

Singapore Campus

100 Victoria Street

National Library Building \# 13-02

Singapore 188064

essecasia@essec.fr

Tél. +6568849780

Fax +6568849781

www.essec.edu

\section{Informations}

Alison Bougi

+33 (0)134433358

bougi@essec.edu

unw.essec.fr

research.center@essec.fr

ISSN 1291-9616 